\title{
THE SPITZER-IRAC/MIPS EXTRAGALACTIC SURVEY (SIMES) IN THE SOUTH ECLIPTIC POLE FIELD
}

\author{
I. Baronchelli ${ }^{1,2}$, C. Scarlata ${ }^{1}$, G. Rodighiero ${ }^{2}$, A. Franceschini ${ }^{2}$, P. L. Capak ${ }^{3}$, S. Mei $^{3,4,5}$, M. Vaccari ${ }^{6,7}$, L. Marchetti ${ }^{8}$, \\ P. Hibon $^{9}$, C. Sedgwick ${ }^{8}$, C. Pearson ${ }^{8,10,11}$, S. Serjeant ${ }^{8}$, K. Menéndez-Delmestre ${ }^{12}$, M. Salvato ${ }^{13}$, M. Malkan ${ }^{14}$, \\ H. I. Teplitz ${ }^{3}$, M. Hayes $^{15}$, J. Colbert ${ }^{3}$, C. Papovich ${ }^{16}$, M. Devlin ${ }^{17}$, A. Kovacs ${ }^{1,3}$, K. S. ScotT ${ }^{18}$, J. Surace ${ }^{3}$, \\ J. D. Kirkpatrick ${ }^{3}$, H. Atek ${ }^{19}$, T. Urrutia $^{20}$, N. Z. Scoville $^{3}$, and T. T. Takeuchi ${ }^{21}$ \\ ${ }^{1}$ MN Institute for Astrophysics, University of Minnesota, 116 Church Street SE, Minneapolis, MN 55455, USA \\ ${ }^{2}$ Dipartimento di Fisica e Astronomia, Università di Padova, vicolo Osservatorio, 3, I-35122 Padova, Italy \\ ${ }^{3}$ California Institute of Technology, 1200 E. California Boulevard, Pasadena, CA 91125, USA \\ ${ }^{4}$ GEPI, Observatoire de Paris, PSL Research University, CNRS, University of Paris Diderot, 61, Avenue de l'Observatoire F-75014, Paris, France \\ ${ }^{5}$ University of Paris Denis Diderot, University of Paris Sorbonne Cité (PSC), F-75205 Paris Cedex 13, France \\ ${ }^{6}$ Astrophysics Group, Physics Department, University of the Western Cape, Private Bag X17, 7535 Bellville, Cape Town, South Africa \\ INAF-Istituto di Radioastronomia, via Gobetti 101, I-40129 Bologna, Italy \\ ${ }^{8}$ Department of Physical Sciences, The Open University, Milton Keynes, MK7 6AA, UK \\ ${ }^{9}$ Gemini South Observatory, Casilla 603, La Serena, Chile \\ ${ }^{10}$ RAL Space, Rutherford Appleton Laboratory, Chilton, Didcot, Oxfordshire OX11 0QX, UK \\ ${ }^{11}$ Oxford Astrophysics, Denys Wilkinson Building, University of Oxford, Keble Road, Oxford OX1 3RH, UK \\ ${ }^{12}$ Observatório do Valongo, Universidade Federal de Rio de Janeiro, Rio de Janeiro, Brazil \\ ${ }^{13}$ Max Planck institute for extraterrestrial Physics, Giessenbachstrasse 1, Garching, D-85748, Germany \\ ${ }^{14}$ Department of Physics and Astronomy,UCLA, Physics and Astronomy Building, 3-714, LA CA 90095-1547, USA \\ ${ }^{15}$ Department of Astronomy, Oskar Klein Centre, Stockholm University, AlbaNova University Centre, SE-106 91 Stockholm, Sweden \\ ${ }^{16}$ Department of Physics and Astronomy, Texas A\&M University, College Station, TX 77843-4242, USA \\ ${ }^{17}$ Department of Physics and Astronomy, University of Pennsylvania, 209 South 33rd Street, Philadelphia, PA 19104, USA \\ ${ }^{18}$ National Radio Astronomy Observatory, 520 Edgemont Road, Charlottesville, VA 22903, USA \\ ${ }^{19}$ Laboratoire d'Astrophysique, Ecole Polytechnique Fédérale de Lausanne, Observatoire de Sauverny, CH-1290 Versoix, Switzerland \\ ${ }^{20}$ Leibniz Institut für Astrophysik Potsdam, An der Sternwarte 16, D-14482 Potsdam, Germany \\ ${ }^{21}$ Division of Particle and Astrophysical Science, Nagoya University, Furo-cho, Chikusa-ku, Nagoya 464-8602, Japan \\ Received 2015 September 18; accepted 2016 January 2; published 2016 March 7
}

\begin{abstract}
We present the Spitzer-IRAC/MIPS Extragalactic survey (SIMES) in the South Ecliptic Pole field. The large area covered $\left(7.7 \mathrm{deg}^{2}\right)$, together with one of the lowest Galactic cirrus emissions in the entire sky and a very extensive coverage by Spitzer, Herschel, Akari, and GALEX, make the SIMES field ideal for extragalactic studies. The elongated geometry of the SIMES area $(\approx 4: 1)$, allowing for significant cosmic variance reduction, further improves the quality of statistical studies in this field. Here we present the reduction and photometric measurements of the Spitzer/IRAC data. The survey reaches depths of 1.93 and $1.75 \mu \mathrm{Jy}(1 \sigma)$ at 3.6 and $4.5 \mu \mathrm{m}$, respectively. We discuss the multiwavelength IRAC-based catalog, completed with optical, mid-, and far-IR observations. We detect 341,000 sources with $F_{3.6 \mu \mathrm{m}} \geqslant 3 \sigma$. Of these, $10 \%$ have an associated $24 \mu \mathrm{m}$ counterpart, while $2.7 \%$ have an associated SPIRE source. We release the catalog through the NASA/IPAC Infrared Science Archive. Two scientific applications of these IRAC data are presented in this paper. First, we compute integral number counts at $3.6 \mu \mathrm{m}$. Second, we use the [3.6]-[4.5] color index to identify galaxy clusters at $z>1.3$. We select 27 clusters in the full area, a result consistent with previous studies at similar depth.
\end{abstract}

Key words: catalogs - galaxies: evolution - infrared: galaxies - submillimeter: galaxies - surveys

\section{INTRODUCTION}

Contrary to the expectation that galaxy formation would proceed via merger-driven bursts of star formation (SF), evidence is now overwhelmingly showing that the bulk of SF in the universe happened in a "quiescent" mode, at increasingly higher average rates at earlier cosmic times (e.g., Daddi et al. 2007; Noeske et al. 2007; Peng et al. 2010; Rodighiero et al. 2011). Although short-lived powerful merger-driven starbursts $\left(\mathrm{SFR}>1000 \mathrm{M}_{\odot} \mathrm{yr}^{-1}\right.$ ) do not contribute significantly to the slow process of galaxy growth, they may represent a critical phase in the structural transformation and quenching of the most massive galaxies (e.g., Rodighiero et al. 2011; Bedregal et al. 2013).

The most actively SF galaxies at any redshift also tend to be the most dust-obscured objects. They disappear at rest-frame UV wavelengths and emit most of their energy in the far-IR where they can be easily identified through imaging between
24 and $500 \mu \mathrm{m}$. The Herschel satellite with its PACS and SPIRE instruments (Griffin et al. 2010; Pilbratt et al. 2010; Poglitsch et al. 2010) have revolutionized the field, producing large samples of mid-IR bright galaxies, selected up to very large redshifts via their bolometric luminosity (e.g., Rodighiero et al. 2010, 2011; Wuyts et al. 2011; Magnelli et al. 2012; Oliver et al. 2012; Rosario et al. 2012; Gruppioni et al. 2013; Santini et al. 2014). To understand the physical nature of these sources, however, detailed sampling of the full spectral energy distribution (SED) and (spectroscopic/photometric) redshifts are needed, and require a secure counterpart association at shorter wavelengths. With secure counterparts and sufficient ancillary data, accurate photometric redshifts can be computed that will allow the effective use of the Atacama Large Millimeter/submillimeter Array, not only to measure spectroscopic redshifts, but also to understand the physical conditions of the molecular gas reservoir in these objects. 
In this paper, we present the Spitzer-IRAC/MIPS Extragalactic survey (SIMES), an infrared survey carried out with the Spitzer Space telescope (Fazio et al. 2004b; Werner et al. 2004) in a $7.7 \mathrm{deg}^{2}$ field close to the South Ecliptic Pole (SEP) at 3.6 and $4.5 \mu \mathrm{m}$. The SIMES field, centered at $(\alpha$, $\delta)=\left(4^{\mathrm{h}} 44^{\mathrm{m}},-53^{\circ} 30^{\prime}\right)$, has Galactic cirrus emission among the lowest in the entire sky $\left(\sim 2-3 \mathrm{MJy} \mathrm{str}^{-1}\right.$ at $100 \mu \mathrm{m}$, Schlegel et al. 1998; Matsuhara et al. 2006), thus minimizing the extinction in the UV and optical bands as well as maximizing the sensitivity at far-IR wavelengths. This field is therefore very appealing for full multiwavelength exploitation. Furthermore, it has the unique advantage of having an elongated geometry (axial ratio of approximately $\sim 4: 1$ ), which minimizes the cosmic variance compared to square fields of similar depth and area on the sky (Trenti \& Stiavelli 2008).

The SIMES field has been the target of a vast array of multiwavelength observing programs from major observatories: Spitzer (Clements et al. 2011), Herschel (Oliver et al. 2012; Wang et al. 2014), GALEX (Dale et al. 2007). In particular, together with the North Ecliptic Pole area, it is one of the two fields including the deepest contiguous observations by the Akari IR observatory in the context of the AKARI Deep Field South survey (Matsuhara et al. 2006; Matsuura et al. 2011). These observations provide us with the most extensive photometric coverage in the mid-IR available for cosmological surveys, of particular relevance for the analysis of dust-obscured active galaxies and AGNs. Very important for the identification at IR and optical wavelengths is the availability of Spitzer $24 \mu \mathrm{m}$ imaging (Rieke et al. 2004), which, with its 5" 0 beam, nicely links imaging at shorter and longer wavelengths. Until our survey, however, the SIMES field was missing the crucial IRAC coverage required to associate the majority of broad-beamed/confused $\geqslant 24 \mu \mathrm{m}$ sources with physically understood astrophysical objects (detected at $\lambda<1 \mu \mathrm{m}$ ). Here we present the new IRAC observations of the SIMES field, targeting this outstanding wavelength gap and thus allowing the full exploitation of the available longer wavelength data. These data will also be crucial for the measurement of physical properties of highredshift objects, including their photometric redshifts and stellar masses.

The paper is organized as follows. We describe the IRAC observations and catalog preparation in Section 2. The matching with the long wavelength ancillary data is described in Section 3. Finally, we discuss initial results on the $3.6 \mu \mathrm{m}$ number counts and the identification of intermediate-redshift galaxy clusters in Section 4. Throughout this paper, we assume a standard flat cosmology with $H_{0}=70 \mathrm{Km} \mathrm{s}^{-1} \mathrm{Mpc}^{-1}$, $\Omega_{M}=0.3$, and $\Omega_{\Lambda}=0.7$. Wherever magnitudes or colors are reported, the $\mathrm{AB}$ magnitude system is implicitly assumed.

\section{OBSERVATIONS AND DATA ANALYSIS}

SIMES is a Spitzer Cycle 8 General Observer program (PID 80039, P.I.: Scarlata) observed during the warm mission phase. The SIMES survey in the IRAC bands was designed with the goal of complementing the existing MIPS $24 \mu \mathrm{m}$ and far-IR observations. The $7.74 \mathrm{deg}^{2}$ field was covered in $78 \mathrm{hr}$ with the IRAC instrument in both channels 1 and 2, corresponding to imaging at $3.6 \mu \mathrm{m}$ and $4.5 \mu \mathrm{m}$, respectively. In order to efficiently cover the elongated region, we used multiple $4 \times 16$ IRAC AORs. This strategy was chosen to minimize the effect of the substantial rotation (1 deg per day) at the field latitude.
The field was covered in two visits, between 2011 November 16 and 23 , in order to facilitate identification and removal of asteroids. The first and second visits consisted of $3 \times 30 \mathrm{~s}$ and $2 \times 30$ s frames, respectively, obtained with a medium cycling dither pattern, for a total exposure time of $150 \mathrm{~s}$. The reduction of the IRAC data generally followed the procedure used by the Spitzer Enhanced Imaging Products (SEIP) mosaic pipeline (Capak et al. 2013) with one additional step. Before the SEIP mosaic processing, a median image was created for each AOR (observing block) and subtracted from the frames to remove residual bias in the frames and persistence from previous observations. The MOPEX Overlap routine (Makovoz et al. 2005) was then used on the background subtracted images to remove any residual background variation from frame to frame. The median subtracted frames were then combined with the MOPEX mosaic pipeline (Makovoz \& Khan 2005). The outlier and box-outlier modules were used to reject cosmic rays, transients, and moving objects. Many of the exposures were affected by latent images from prior observations of a bright object, although the impact was much more severe in channel 1 than in channel 2 where it effectively doubled the noise over the background-limited estimate. The effects of these latent images could not be fully mitigated because they faded during the observations, and so a perfect model could not be produced. As a result, the $3.6 \mu \mathrm{m}$ data have a sensitivity comparable to the $4.5 \mu \mathrm{m}$ data (see Section 2.2 and Table 2). As a final step, the data were then interpolated onto a 0 " 6 pixel scale using a linear interpolation and combined with an exposure time weighted mean combination. Mean, median, coverage, uncertainty, and standard-deviation images were created. The final resulting mosaic is shown in the top panel of Figure 1, while the bottom panel shows a comparison of the IRAC coverage with the coverage in the MIPS, SPIRE, and optical surveys within the same area.

\subsection{Source Extraction and Photometry}

For the detection and extraction of sources we used the SExtractor software (Bertin \& Arnouts 1996) in dual image mode, using the $3.6 \mu \mathrm{m}$ map as the detection image and the uncertainty map as a weight image. During the detection step, we used a local background calculated over an area of $32 \times 32$ pixels filtered with a 3 pixel top-hat kernel. We set a $1.5 \sigma$ threshold, with a minimum of 5 connected pixels above the background noise. For each object we computed AUTO total fluxes, as well as aperture fluxes measured in apertures of 4 " 8,7 ". 2 , and 12 !" 0 diameter.

SExtractor AUTO fluxes are estimates of the total flux of a source in an elliptical aperture with semimajor axis (a) proportional to the Kron radius of the object $\left(R_{K}\right.$, Kron 1980). We chose $a=2.5 R_{K}$ by setting the SExtractor parameter Kron_fact $=2.5{ }^{22}$ This choice ensures that the aperture includes more than the $90 \%$ of the total galaxy flux ${ }^{23}$ (Kron 1980). For apertures with $a<3.5$ pixels (2"' 1 ), the AUTO flux is computed within a circular aperture. AUTO fluxes account for the real apparent dimension of each source,

\footnotetext{
22 In SExtractor nomenclature, $a=$ KRON_RADIUS $\times$ A_IMAGE, where A_IMAGE is the luminosity profile rms, in pixels, along the major axis direction, while KRON_RADIUS $=$ Kron_fact $\times R_{K}$, with $R_{K}$ in units of A_IMAGE.

${ }^{23}$ The Kron aperture includes a different fraction of the total light of a galaxy, depending on the value of the Sérsic index $n$ of its surface brightness profile (Sérsic 1963; Graham \& Driver 2005).
} 

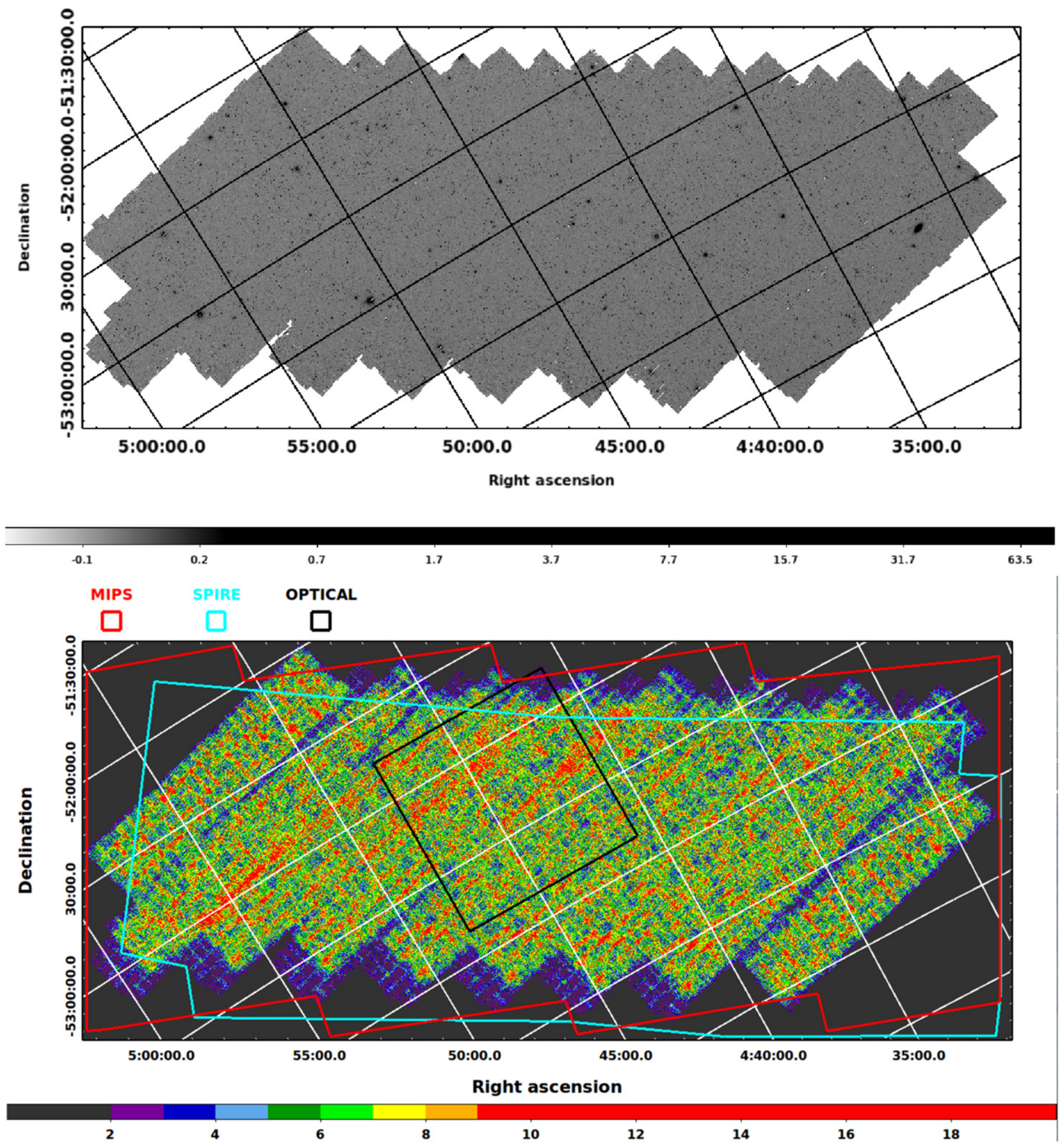

Figure 1. Top panel: IRAC $3.6 \mu \mathrm{m}$ mosaic of the SIMES field. The color scale is in units of $\mathrm{MJy} \mathrm{sr}^{-1}$. Bottom panel: Coverage map at $3.6 \mu \mathrm{m}$. The color scale shows the number of frames per pixel. The areas covered by MIPS, SPIRE, and WFI- $R_{c}$ (optical) are shown in red, cyan, and black, respectively.

the elliptical shape of the observed isophotes and the source's radial surface brightness profile.

In Figure 2, we report the mean ratio $\langle R\rangle$ between the AUTO fluxes and the APERTURE fluxes computed for our sources in bins of semimajor axis. $\langle R\rangle$ increases with the increasing apparent dimension of the sources, indicating that a fraction of the sources' emission is missed when using a fixed aperture.
There is a strong linear correlation between $\log \langle R\rangle$ and the semimajor axis of the elliptical aperture, with different slopes depending on the size of the circular aperture used. The ratio becomes $\sim 1$ when the semimajor axis of the elliptical aperture has a dimension similar to that of the circular aperture used for the comparison. When considering the smallest elliptical apertures and the largest circular apertures, we observe a large 


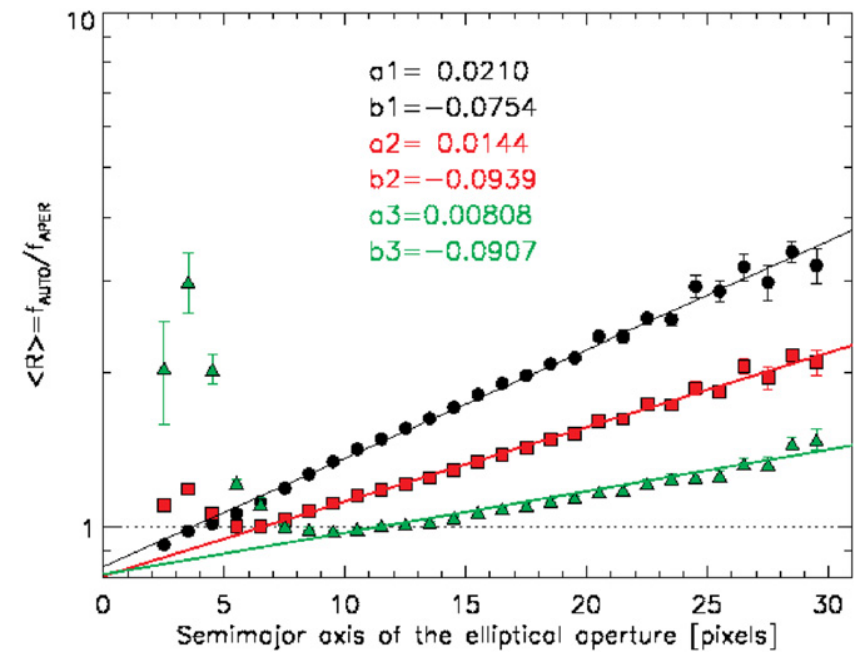

Figure 2. Average ratio between AUTO fluxes and uncorrected aperture fluxes ( 4 . 8 in black, $7 "$. 2 in red, 12 ". 0 in green), as a function of the semimajor axis of the Kron elliptical aperture, together with best linear fit coefficients, expressed as $\log \langle R\rangle=a \times$ (semimajor axis) $+b$. The figure shows how an increasing amount of the source's emission is missed when using fixed circular apertures to compute fluxes.

deviation from $\langle R\rangle \sim 1$. This effect is likely due to an overestimate of the background that becomes appreciable when the elliptical and circular apertures have very different sizes. Sources characterized by small apparent dimensions (i.e., small semimajor axis of the elliptical aperture) tend to have smaller aperture fluxes for larger aperture sizes (green curves in Figure 2). Thus, hereafter, all fluxes reported are total fluxes "FLUX_AUTO" measured within the Kron SExtractor apertures.

\subsection{Survey Sensitivity}

The mapping strategy adopted to cover the large SIMES area results in varying coverage across the field with a resulting noise variation. Figure 3 shows the cumulative distribution of the pixel coverage in the $3.6 \mu \mathrm{m}$ mosaic, where the coverage on the horizontal axis is defined as the number of $30 \mathrm{~s}$ exposures per pixel. More than $70 \%$ of the mosaic is at or above the planned coverage of $150 \mathrm{~s}$.

In order to identify reliable detections in the $3.6 \mu \mathrm{m}$ catalog, we follow Surace et al. (2005) and compute a coverage-based signal-to-noise ratio $(\mathrm{S} / \mathrm{N})$ for each source. Surace et al. (2005) compute the noise $\sigma$ corresponding to the mean coverage within the mosaic $(\langle C\rangle$, where $C$ is the number of exposures per pixel) and then scale it by a factor $f$ that accounts for the specific coverage, $C$, of the aperture used for the flux measurement, i.e., $f=\sqrt{\langle C\rangle / C}$. This procedure assumes that the noise scales as the square root of the exposure time. However, the noise contribution from faint unresolved sources could be substantial in the deepest regions of the mosaic.

In order to check the $t_{\exp }^{-0.5}$ assumption, we empirically derived the noise properties of the mosaic as a function of the actual coverage. First, we measured the flux in 8 pixel diameter apertures distributed in a homogeneous grid covering the mosaic. Then, we divided our measures in different groups, according to the coverage underlying the apertures in which they were obtained. In each bin of coverage, we fitted a Gaussian function to the distribution of aperture fluxes, symmetrized with respect to the median to include only

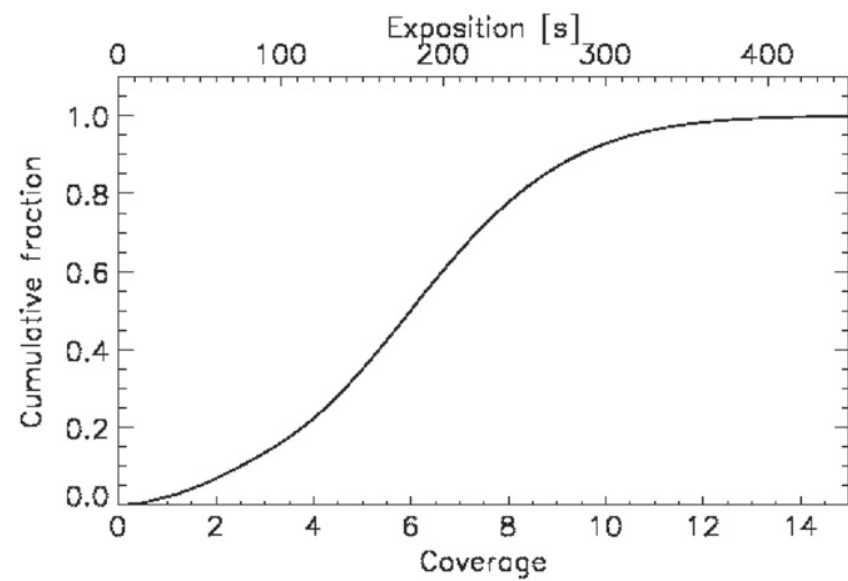

Figure 3. Cumulative distribution of the pixel coverage. More than $70 \%$ of the pixels in the mosaic are at the nominal $150 \mathrm{~s}$ coverage.

background-dominated apertures. The standard deviation of the best-fit Gaussian distribution in each bin of coverage is then a measurement of the average background noise corresponding to that coverage.

To check the normal distribution of the pixels noise, we applied both the Kolmogorov-Smirnov (K-S) and AndersonDarling (A-D) tests to the negative side of the pixel flux distribution. Because the noise level is expected to depend on the exposure time, we consider seven equally sized bins of coverage ranging between 2 and 10 . At the nominal coverage of 5, using the $\mathrm{K}-\mathrm{S}$ test, the probability of finding the computed difference $D=3.9 \times 10^{-3}$ between the cumulative distribution of fluxes and that expected from a normal distribution is 0.74 . Using the A-D test, we found a difference of $A^{2}=0.55$, which is close to the reference value of 0.576 , corresponding to the rejection of the null hypothesis (normality) with a $15 \%$ level of significance. At lower and higher coverages, the probability from the $\mathrm{K}-\mathrm{S}$ test ranges from 0.28 to 0.96 , while $A^{2}$ ranges from 0.49 to 1.87 , indicating that when a deviation from the normal distribution is present, it is small.

In Figure 4, we show the resulting $\sigma$ as a function of average coverage $\langle C\rangle$ in each of the six bins. The dependency with $C$ is in agreement with the Poissonian approximation (shown with a continuous red line). The theoretical curve is normalized at the nominal coverage of the survey $(C=5)$. The trend between $\sigma$ and $C$ is well reproduced by the relation $\sigma \propto C^{-\alpha}$, with $\alpha=0.43 \pm 0.09$ (green dashed line in Figure 4). To calculate the $\mathrm{S} / \mathrm{Ns}$, we computed APERTURE flux and noise in the same aperture ( 4 " 8 diameter). For each source, the underlying coverage is computed as the median value in the aperture. We retain in the final catalog only sources with $3.6 \mu \mathrm{m}$ flux above $3 \sigma$. The final IRAC-based catalog constructed in this way includes 341,006 sources.

We compute the sensitivity of the $4.5 \mu \mathrm{m}$ observations using the same method described for the $3.6 \mu \mathrm{m}$ data. Again, measuring $\sigma$ as a function of the average coverage, we found an agreement with the Poissonian expectation, with $\alpha$ $(4.5 \mu \mathrm{m})=0.53 \pm 0.08$.

At all coverages, we found a $4.5 \mu \mathrm{m}$ depth $\sigma$ comparable to or smaller than that measured at $3.6 \mu \mathrm{m}$. In particular, at the nominal coverage of the survey, we measure $\sigma=1.93 \mu \mathrm{Jy}$ at $3.6 \mu \mathrm{m}$ and $\sigma(C=5)=1.75 \mu \mathrm{Jy}$ at $4.5 \mu \mathrm{m}$. As noted before, 


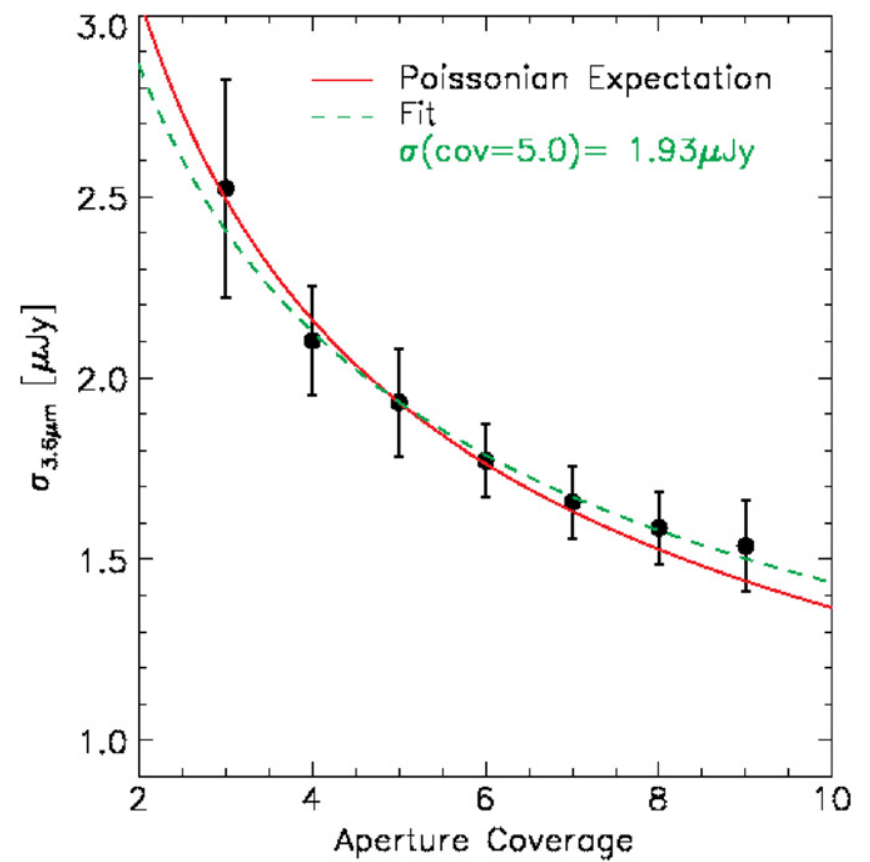

Figure 4. Sky background noise, $\sigma$, as a function of aperture coverage (see the text for details). The expected trend for background-dominated noise is shown with a red solid line, while the observed best-fit relation is shown with a green dashed line. We cut the IRAC $3.6 \mu \mathrm{m}$ catalog at a $3 \sigma$ level, where $\sigma$ is estimated from the average coverage of each source.

this behavior is due to the effect of latent images being more pronounced in the $3.6 \mu \mathrm{m}$ channel than in the $4.5 \mu \mathrm{m}$ channel.

\subsection{Survey Completeness and Contamination}

We estimated the survey completeness as a function of the $3.6 \mu \mathrm{m}$ flux, adding artificial sources to the original IRAC mosaic and extracting them with the same procedure used for the real IRAC map. To create the artificial sources, we generated a synthetic point-spread function (PSF) using the median of 76 images of point sources extracted from the original $3.6 \mu \mathrm{m}$ map. These sources were selected for being isolated (closest counterpart distance $>22$ !" 0 ) and with fluxes near $100 \mu \mathrm{Jy}$. Moreover, we excluded clearly extended sources, characterized by KRON_RADIUS $\times$ A_IMAGE $>10$ pixels and sources located at the edge of the map.

We simulated approximately 69,000 artificial sources with 31 different $3.6 \mu \mathrm{m}$ fluxes in the range $\sim 3-100 \mu \mathrm{Jy}$. For each of the 31 groups, we simulated an independent IRAC map, randomly distributing 2233 same-flux artificial sources along with the real ones. After the extraction, we computed the detection rate (i.e., the completeness) as the ratio between the number of sources inserted in the map and the recovered ones. This approach allows us to maximize the number of sources inserted in the maps without artificially increasing the spatial density of the sources, as would happen if we added all the simulated sources at once. The results of this analysis are presented in Figure 5, where we show that the completeness drops below $50 \%$ at $3.6 \mu \mathrm{m}$ flux of approximately $9.0 \mu \mathrm{Jy}$ (corresponding to a source flux of approximately $4.7 \sigma$ ).

We also investigate the flux accuracy as a function of the artificial source flux by computing the average difference between the flux of the simulated sources and their flux after the extraction. The results, presented in the bottom panel of
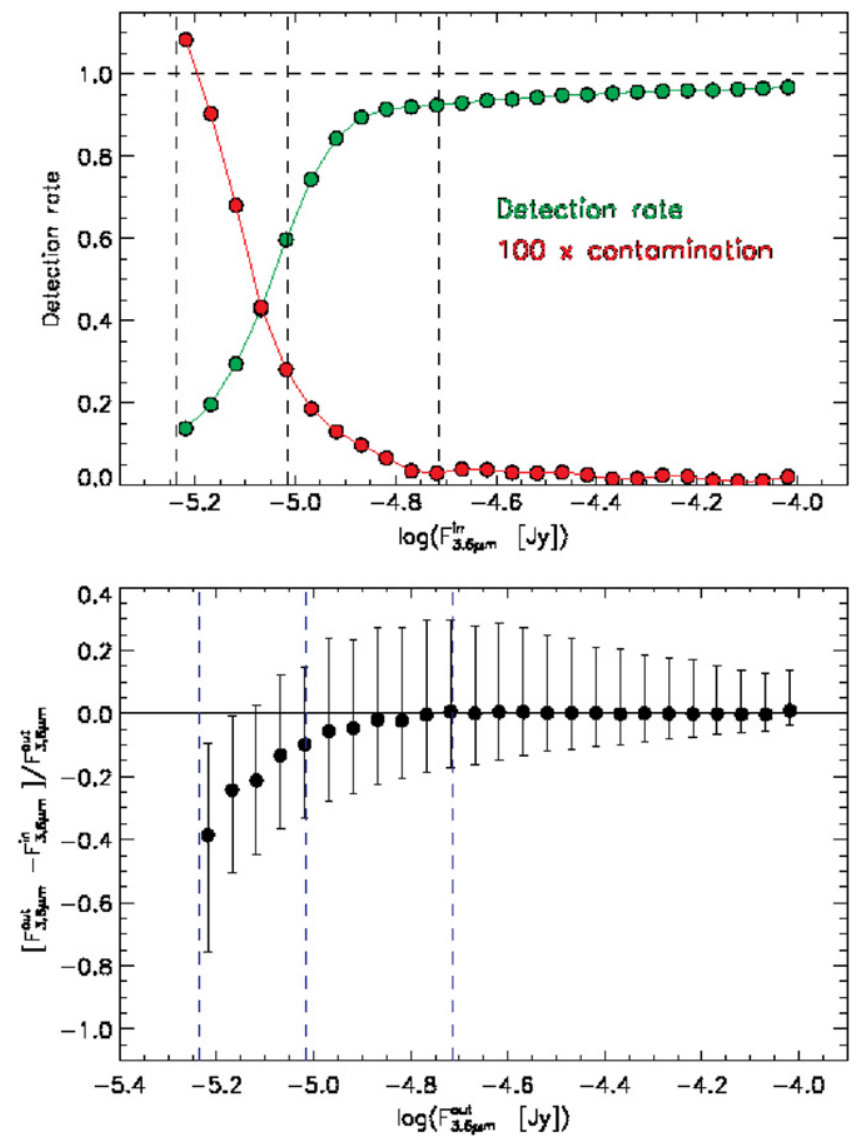

Figure 5. Top panel: completeness and contamination computed through numerical simulations. The completeness level drops to approximately $50 \%$ at $3.6 \mu \mathrm{m}$ flux $\sim 9 \mu \mathrm{Jy}$. The contamination rate (multiplied by a factor of 100 , for clarity) reaches a maximum of $1.1 \%$ at the $3 \sigma$ flux. Bottom panel: Flux accuracy of the simulated sources. The error bars represent the $1 \sigma$ range of flux accuracy resulting from the simulations. Source fluxes start to become systematically underestimated (by more than $10 \%$ ) below $\sim 9 \mu \mathrm{Jy}$. The vertical dashed lines show our 3,5 , and $10 \sigma$ flux limits.

Table 1

Completeness as a Function of $3.6 \mu \mathrm{m}$ Flux

\begin{tabular}{lc}
\hline \hline $\begin{array}{l}\text { Completeness } \\
(\%)\end{array}$ & $\begin{array}{c}\text { Flux } 3.6 \mu \mathrm{m} \\
(\mu \mathrm{Jy})\end{array}$ \\
\hline 50 & 9.0 \\
75 & 10.8 \\
90 & 14.1 \\
95 & 40.0 \\
97 & 107.1 \\
99 & 224.2 \\
\hline
\end{tabular}

Figure 5, show that the accuracy of the flux measurements is a function of the output fluxes. For sources at a $3 \sigma$ level, the recovered flux ranges ( $1 \sigma$ of the data distribution) from $\sim 75 \%$ to $\sim 10 \%$ below the input flux, while for sources at the $10 \sigma$ level the range is from $\sim 20 \%$ below to $\sim 30 \%$ above the input flux. There is an indication that faint sources $\left(F_{3.6 \mu \mathrm{m}}<5 \sigma\right)$ have systematically underestimated fluxes, although the scatter in this range is also larger. The detection rate as a function of the $3.6 \mu \mathrm{m}$ flux is summarized in Table 1 . These values have been used for computing the $3.6 \mu \mathrm{m}$ number counts presented in Section 4.1. 
Table 2

Available Ancillary Data

\begin{tabular}{|c|c|c|c|c|c|c|c|}
\hline \multirow{2}{*}{$\begin{array}{l}\text { Band } \\
(\mu \mathrm{m})\end{array}$} & \multirow{2}{*}{ Instrument } & \multirow{2}{*}{$\begin{array}{l}\text { Overlap Area } \\
{ }_{\left(\mathrm{deg}^{2}\right)}\end{array}$} & \multirow{2}{*}{ Depth } & \multicolumn{4}{|c|}{ Number of Identified Counterparts ${ }^{\mathrm{b}}$} \\
\hline & & & & All & MIPS 24 & SPIRE & MIPS 24 and SPIRE \\
\hline 3.6 & IRAC & 7.74 & $5.80 \mu \mathrm{Jy}(3 \sigma)^{\mathrm{c}, \mathrm{d}}$ & 341006 & 25132 & 9447 & 7041 \\
\hline 4.5 & IRAC & 7.26 & $5.25 \mu \mathrm{Jy}(3 \sigma)^{\mathrm{d}}$ & 320460 & 23688 & 9320 & 6947 \\
\hline 24 & MIPS & 7.66 & $0.26 \mathrm{mJy}(50 \% \text { compl. })^{\mathrm{e}}$ & $25132(60)$ & $25132(60)$ & $7041(60)$ & $7041(60)$ \\
\hline 70 & MIPS & 7.66 & 24 mJy $(50 \% \text { compl. })^{\mathrm{e}}$ & 882 & 882 & 692 & 692 \\
\hline 250 & SPIRE & 6.52 & $15.6 \mathrm{mJy}(3 \sigma)^{\mathrm{f}}$ & $8743(50)$ & $6666(50)$ & $8743(50)$ & $6666(50)$ \\
\hline 350 & SPIRE & 6.52 & $12.7 \mathrm{mJy}(3 \sigma)^{\mathrm{f}}$ & $9416(60)$ & $7015(60)$ & $9416(60)$ & $7015(60)$ \\
\hline 500 & SPIRE & 6.52 & $18.5 \mathrm{mJy}(3 \sigma)^{\mathrm{f}}$ & $8624(58)$ & $6354(58)$ & $8624(58)$ & $6354(58)$ \\
\hline 0.65 & WFI & 1.13 & $0.53 \mu \mathrm{Jy}(3 \sigma)$ & 27585 & 2279 & 808 & 680 \\
\hline
\end{tabular}

Notes.

a Area covered in both the IRAC $3.6 \mu \mathrm{m}$ band and in the band indicated in the first column.

$\mathrm{b}$ The additional number of MIPS-SPIRE sources without $3.6 \mu \mathrm{m}$ counterparts is indicated in parenthesis.

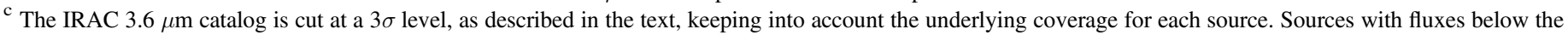
value reported in the table can consequently be found in the catalog.

$\mathrm{d}$ The value of $\sigma$ reported is estimated for the nominal coverage of the survey $(C=5)$.

e From Clements et al. (2011). Minimum $24 \mu \mathrm{m}$ flux in the catalog: $0.20 \mathrm{mJy}$. Minimum for MIPS identified sources with a SPIRE counterpart: $0.31 \mathrm{mJy}$.

f $1 \sigma$ values from Oliver et al. (2012). We included in our catalog only the sources with a flux higher then $3 \sigma$ in at least one of the SPIRE bands.

We estimate the false positive rate (i.e., contamination rate) applying the same extraction technique and $3 \sigma$ cut used for the original $3.6 \mu \mathrm{m}$ map, to the inverted $3.6 \mu \mathrm{m}$ image (pixel fluxes multiplied by -1 ). We obtain a total contamination rate of $0.21 \%$. In Figure 5, the contamination (multiplied for a factor of 100) is shown as a function of the flux of the spurious extracted sources. Spurious sources represent $\sim 1.1 \%$ of all sources at the $3 \sigma$ level and $\sim 0.25 \%$ at $5 \sigma$. In order to obtain an independent upper limit to the total contamination rate, we apply the B-H test (Benjamini \& Hochberg 1995) under the assumption that the background distribution is known and Gaussian. For each flux in the catalog we can then compute its $p$-value under the null hypothesis that it was extracted from the background population. The $p$-value relative to the $3 \sigma$ flux threshold adopted for the $3.6 \mu \mathrm{m}$ catalog $\left(p_{3 \sigma}\right)$ corresponds to the value $C\left(p_{3 \sigma}\right)$ in the $p$-values cumulative distribution. The expected contamination rate $\epsilon=(1-\alpha)$ $N p_{3 \sigma} / C\left(p_{3 \sigma}\right)<N p_{3 \sigma} / C\left(p_{3 \sigma}\right)$, where $N$ is the number of apertures used in the test and $\alpha$ is the ratio between the number of spurious and real sources in the catalog. At the nominal coverage of 5, the upper limit on the contamination rate is then $1.2 \%$.

\section{ANCILLARY DATA}

The SIMES field is fully covered with both Spitzer MIPS (24 and $70 \mu \mathrm{m})$, and Herschel SPIRE (250, 350, and $500 \mu \mathrm{m})$ as well as by Akari. In the present paper we report on the MIPS and SPIRE observations, while the Akari data will be discussed in a forthcoming paper (I. Baronchelli et al. 2016, in preparation). The central square degree is also covered by optical imaging (see Figure 1). In the following sections we describe how we merged the IRAC-based catalog with the publicly available MIPS (Clements et al. 2011) and SPIRE (HerMES, DR2, Roseboom et al. 2010; Oliver et al. 2012; Smith et al. 2012; Wang et al. 2014) catalogs. Section 3.3 describes the data reduction, photometry, and matching of the optical data. The main properties of the multiwavelength data are summarized in Table 2.

\subsection{MIPS 24 and $70 \mu \mathrm{m}$}

The MIPS $24 \mu \mathrm{m}$ catalog is described in Clements et al. (2011). The Clements et al. (2011) catalog covers an area of $\sim 12 \mathrm{deg}^{2}$ in the SEP region and includes counterparts at $70 \mu \mathrm{m}$ of the $24 \mu \mathrm{m}$ detected sources, and so we limit the analysis to the cross-correlation between IRAC and MIPS 24 and report the $70 \mu \mathrm{m}$ association identified in the original MIPS catalog. Clements et al. (2011) estimate that the $24 \mu \mathrm{m}$ catalog is $50 \%$ complete at $0.26 \mathrm{mJy}$ and $80 \%$ complete at $0.32 \mathrm{mJy}$, while the source reliability is $96 \%$ at $0.285 \mathrm{mJy}$.

In order to identify the most likely IRAC counterpart to each MIPS source, we proceed as follows. For each MIPS $24 \mu \mathrm{m}$ source, we searched the IRAC catalog for the nearest object inside a radius equal to the quadratic sum of the $\sigma$ of the PSF of the two instruments (i.e., a search radius of 2!"6). In the matching process, we identified a small systematic shift ${ }^{24}$ (of the order of $\Delta$ R.A. $=0$ " $099, \Delta$ decl. $=0$ ". 49) between the two catalogs. Therefore, we corrected the MIPS positions before searching for the nearest IRAC counterpart. We report in the final catalog both the corrected and the original coordinates of the sources in each band. In Table 3, we report the distance and the average R.A. and decl. shifts of all of the sources matched in the catalog. When multiple IRAC sources were found within the search area (see Figure 7), we associated the closest IRAC object. This happens for 514 MIPS sources which we flag as uncertain identifications ("N_IRAC_MIPS" parameter greater than one). All the other potential IRAC counterparts can be found in the catalog. Of all of the MIPS sources $(25,132$ objects), $98.0 \%$ have a unique IRAC counterpart within a region of 2!"6 radius. MIPS sources without an IRAC counterpart are generally not included in our catalog. The only exception is represented by 60 visually checked sources in the IRAC covered area with a reliable SPIRE counterpart.

\subsection{SPIRE $250,350,500 \mu \mathrm{m}$}

The SIMES field was observed as part of the Herschel Multitiered Extragalactic Survey (HerMES, Oliver et al. 2012; Wang et al. 2014). The second data release of the SPIRE XID catalogs (DR2, Roseboom et al. 2010; Smith et al. 2012; Wang et al. 2014) covers approximately $84 \%$ of the field, and

\footnotetext{
${ }^{24}$ We verified that the shift did not depend on the position in the large mosaic, thus indicating that any distortion in the IRAC mosaic was properly accounted for.
} 
Table 3

Parameters Used in the Counterpart Identification Procedure ${ }^{\mathrm{a}}$

\begin{tabular}{|c|c|c|c|c|c|c|}
\hline \multirow[b]{2}{*}{ Band } & \multirow{2}{*}{$\begin{array}{c}\text { Search } \\
\text { Radius } \\
\left(!^{\prime \prime}\right)\end{array}$} & \multicolumn{2}{|c|}{ Mean Distance ${ }^{b}$} & \multicolumn{2}{|c|}{ Barycentre Position ( $\Delta$ R.A., $\Delta$ decl. $)^{\mathrm{c}}$} & \multirow[b]{2}{*}{$\Delta N$} \\
\hline & & $\begin{array}{c}\text { Before R. } \\
(! !)\end{array}$ & $\begin{array}{c}\text { After R. } \\
(! !)\end{array}$ & $\begin{array}{c}\text { Before R. } \\
(! !)\end{array}$ & $\begin{array}{c}\text { After R. } \\
(! !)\end{array}$ & \\
\hline MIPS & 2.60 & 1.01 & 0.87 & $0.098,0.49$ & $0.010,0.071$ & $+140(0.6 \%)$ \\
\hline SPIRE $^{\mathrm{d}}$ & 8.04 & 3.00 & 3.00 & $0.019,-0.045$ & $0.0035,-0.0040$ & $-1(0.01 \%)$ \\
\hline 2MASS & 2.68 & 0.60 & 0.59 & $0.067,-0.067$ & $0.0061,-0.0059$ & $+7(0.005 \%)$ \\
\hline
\end{tabular}

Notes.

a All the values are computed before the correction for average shift (Before R.) and after the correction (After R.).

b Average distance, in arcseconds, between the IRAC $3.6 \mu \mathrm{m}$ sources and the corresponding counterparts in the other bands.

c Average difference ( $\Delta$ R.A., $\Delta$ decl.) between the IRAC $3.6 \mu \mathrm{m}$ sources and the corresponding counterparts in the other bands.

${ }^{\mathrm{d}}$ Distances refer to the MIPS 24 positions.

includes all sources identified at the $1 \sigma$ level at 250,350 , or $500 \mu \mathrm{m}$. Here, we keep only those sources with fluxes above $3 \sigma$ in at least one SPIRE band.

The large size of the SPIRE PSF (18!" 0 at $250 \mu \mathrm{m})$ prevents us from directly cross-correlating the SPIRE and IRAC catalogs. Instead, we exploited the MIPS $24 \mu \mathrm{m}$ detections as a "bridge" between the two wavelengths, i.e., we searched the SPIRE counterparts given the MIPS 24 prior position. We performed a direct SPIRE-IRAC correlation only when a SPIRE source did not have a MIPS counterpart. As we did for the MIPS-IRAC correlation, before correlating the SPIRE and MIPS counterparts, we corrected the SPIRE coordinates for the small average offset between MIPS and SPIRE positions using the original MIPS coordinates as reference. In our catalog, we report both the original SPIRE (R.A., decl.) coordinates as well as the coordinates corrected to the average SPIRE-MIPS and then MIPS-IRAC shifts.

Given the MIPS positions, we searched the SPIRE counterparts inside a radius of 8!"04 (quadratic sum of the PSF's $\sigma$ of the two instruments). When a single SPIRE source is associated with two (or more) different MIPS sources, we consider both associations. Both the SPIRE and MIPS fluxes are proportional to the total IR luminosity $\left(L_{\mathrm{IR}}\right.$, e.g., Chary \& Elbaz 2001; Elbaz et al. 2011). This is due to the same thermal origin of the radiation emitted in these bands. Given this assumption, when we find multiple MIPS counterparts for a single SPIRE source, the original fluxes in the three SPIRE bands are divided among the MIPS counterparts proportionally to their $24 \mu \mathrm{m}$ flux. This multiple association involves 429 MIPS sources, flagged in our catalog through a N_MIPS_SPIRE parameter greater than 1 . SPIRE-MIPS associations outside the IRAC covered area are not included in our catalog. Using the MIPS 24 prior position, we found 7034 SPIRE counterparts for our IRAC sources.

For the remaining SPIRE sources without a MIPS counterpart, we searched for a direct IRAC-SPIRE association. We found 2413 SPIRE sources with associated IRAC counterparts. These are flagged in the catalog with N_IRAC_SPIRE $>0$ and N_MIPS_SPIRE $\leqslant 0$. The MIPS-undetected SPIRE sources have $24 \mu \mathrm{m}$ flux below the detection threshold in this band. This is because for SPIRE detected sources, the detection rate at $24 \mu \mathrm{m}$ strongly depends on the source redshift. This is due to the typical shape of the SED of far-IR detected sources ${ }^{25}$, usually presenting a bump of emission centered at $\lambda_{\text {R.F. }}^{\text {dust }} \sim 100 \mu \mathrm{m}$ (Kirkpatrick et al. 2012). This bump is due to the thermal emission of dust

\footnotetext{
25 Some examples of typical SEDs for different types of galaxies can be found in, e.g., Polletta et al. (2007).
}
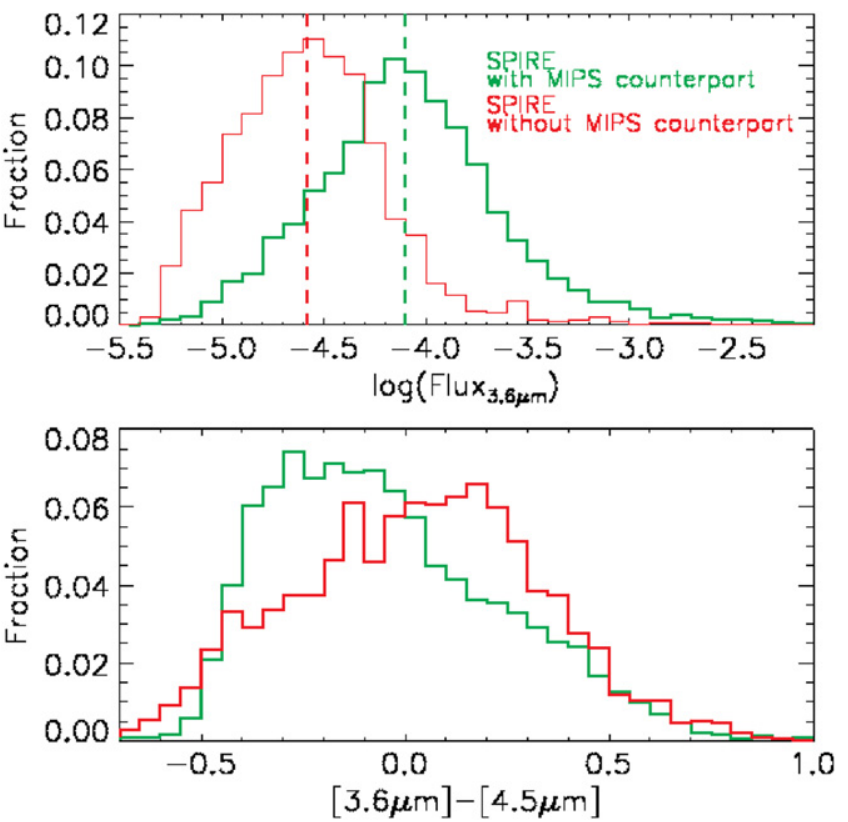

Figure 6. MIPS-undetected SPIRE sources are likely located at higher redshift than MIPS-detected sources. Normalized $3.6 \mu \mathrm{m}$ fluxes (top panel) and [3.6 $\mu \mathrm{m}]-[4.5 \mu \mathrm{m}] \mathrm{AB}$ color distributions (bottom panel) for SPIRE sources in our catalog. The SPIRE sources with and without MIPS counterparts are indicated in green and red, respectively. The median of each distribution is represented with a dashed line in the same color code.

heated by optical-UV radiation produced by young stars inside star forming regions or by accretion disks of active galactic nuclei (AGNs). At increasing redshifts, the SPIRE bands sample spectral regions closer to the redshifted peak. Instead, being located on the opposite side of the thermal emission bump, the MIPS $24 \mu \mathrm{m}$ band samples a spectral region whose the emitted luminosity tends to be lower at higher redshifts. The combination of these effects is responsible for the lower MIPS detection rate among the SPIRE detected sources at higher redshifts. This is demonstrated in Figure 6 where we show the distributions of $3.6 \mu \mathrm{m}$ flux and [3.6 $\mu \mathrm{m}]-[4.5 \mu \mathrm{m}]$ colors (i.e., $\left.2.5 \log \left(F_{4.5} \mu \mathrm{m} / F_{3.6} \mu \mathrm{m}\right)\right)$ for SPIRE sources with and without MIPS counterpart. The two IRAC bands sample the stellar $1.6 \mu \mathrm{m}$ bump (Simpson \& Eisenhardt 1999; Sawicki 2002) and the IRAC color is expected to change with redshift as the peak moves through the two filters. The color distributions in Figure 6 are clearly different, indicating that MIPS-undetected SPIRE sources are more commonly located at higher redshifts than the MIPS-detected SPIRE sources. This is 


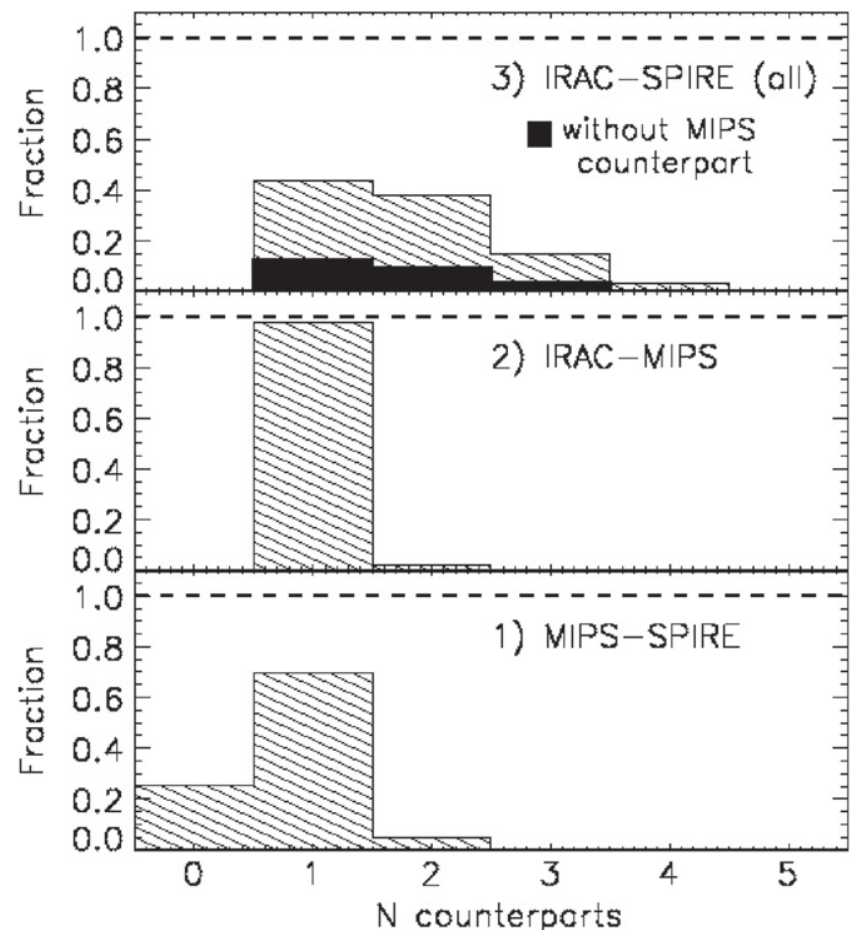

Figure 7. Fraction of sources with one or multiple counterparts inside the search radius for the SPIRE-MIPS, MIPS-IRAC, and SPIRE-IRAC positional correlations. In the top panel, we show the distribution of the number of IRAC counterparts inside the SPIRE-IRAC search aperture for the full sample of SPIRE sources (hatched histogram). The black filled histogram shows the distribution of the number of IRAC counterparts for SPIRE sources without a MIPS counterpart.

also supported by the lower median $3.6 \mu \mathrm{m}$ flux of the MIPSundetected SPIRE sources (upper panel of Figure 6).

SPIRE sources lacking an IRAC counterpart are generally not included in our catalog. However, we include in our catalog the 60 SPIRE sources with a reliable MIPS counterpart but without any IRAC counterpart inside a distance corresponding to two times the IRAC-MIPS search radius. We visually checked all of the sources in the IRAC $3.6 \mu \mathrm{m}$ image in order to exclude missed detections due to the presence of nearby bright sources or border effects enhancing the noise, and consequently, the detection threshold.

The catalog includes a total of 9447 SPIRE sources with a SPIRE flux (in at least one band) above $3 \sigma$. The reliability of their association with the IRAC counterpart is discussed in the next section.

\subsubsection{Counterpart Reliability}

The probability of having the right MIPS (IRAC) counterpart associated with the SPIRE (MIPS) source depends on both the number of galaxies found within the search area, as well as the distance to the identified counterpart(s). In Figure 7 we show the fraction of MIPS and SPIRE sources with one or more IRAC or MIPS counterpart inside the search radius. Only 2\% of our MIPS sources have more than one single IRAC counterpart inside the search radius. In all these cases we consider the closest IRAC source as the only real counterpart. Instead, $\sim 6 \%$ of our SPIRE-MIPS associations are made dividing the SPIRE fluxes among the multiple MIPS counterparts, as explained in Section 3.2. In the direct SPIRE-IRAC associations we considered the closest IRAC sources as the

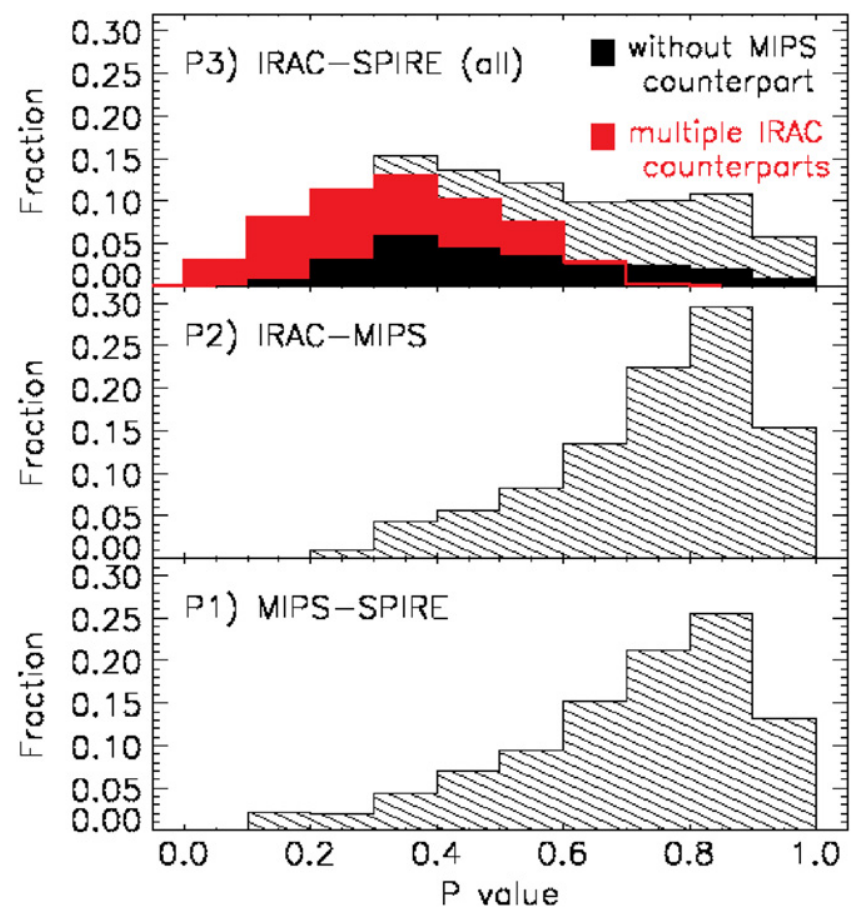

Figure 8. Correlation reliability indicator- $P$-computed for the SPIRE-MIPS $(P 1)$, MIPS-IRAC $(P 2)$, and SPIRE-IRAC $(P 3)$ positional correlations. The latter was computed for all SPIRE sources in our catalog, even when the correlation with IRAC is found through the MIPS position. The black filled distribution in the top panel shows SPIRE sources without a MIPS counterpart. The red filled distribution is computed for SPIRE sources with multiple IRAC counterparts inside the IRAC-SPIRE search radius.

only real counterparts, but in this case, only $\sim 48 \%$ of our SPIRE source have a single IRAC counterpart inside the searhing radius. In order to assess the reliability of the matched counterparts, we develop a parameter, $P$, that accounts for both the counterpart distance and the number of sources found in the search radii.

As mentioned earlier, we define the search area by the radius $r_{s}$ equal to the the quadratic sum of the PSF's $\sigma$ of the two instruments involved (e.g., $r_{s}=\sqrt{\sigma_{\text {IRAC }}^{2}+\sigma_{\text {MIPS }}^{2}}$, for IRACMIPS correlation). We consider a normalized 2D Gaussian function with $\sigma=r_{s}$; the counterpart distance $d_{i}$ is always smaller then $r_{s}$. We then compute the quantity $A_{i}$ as the probability of the galaxy being at a distance greater than $d_{i}$, for the given Gaussian function. For a single counterpart, centered on the coordinates of the starting objects, $A_{i}=1$; in general, $A_{i}$ decreases as the distance of the counterpart increases. We then account for the presence of multiple counterparts (at different $d_{i}$ ), by defining the parameter $P$ as follows:

$$
P=A_{1} \frac{A_{1}}{\sum_{i} A_{i}},
$$

where $A_{1}$ is defined as $A_{i}$ corresponding to the closest counterpart. For multiple counterparts the factor $A_{1} / \sum A_{i}$ is smaller than one, and decreases with the number of counterparts.

We computed the values of $P$ for the SPIRE-MIPS $(P 1)$, MIPS-IRAC $(P 2)$, and SPIRE-IRAC (P3) correlations. The distributions of the $P$ values are represented in Figure 8. The direct SPIRE-IRAC correlation is studied for the whole SPIRE 
sample in our catalog, considering also the sources for which we found a correlation through the MIPS position.

The MIPS-IRAC and SPIRE-MIPS association reliability is usually high, as demonstrated by the distribution of $P 1$ and $P 2$. As a consequence, the association of the SPIRE sources with the IRAC counterparts through the MIPS position is still reliable, even if the $P 3$ distribution is not as narrow as those of $P 1$ and $P 2$.

Using a Monte Carlo simulation, we assessed the reliability of those associations in which multiple MIPS counterparts are associated with individual SPIRE sources (see Section 3.2). The same simulation also allows us to determine the reliability of the direct SPIRE-IRAC associations. The simulation is performed by first randomly shifting the position of the SPIRE sources to 10,20,30, and 50 times the search radius from the original position and then looking for potential IRAC counterparts. We found a detection rate of approximately $49.7 \%$ $\pm 0.1 \%$.

In our catalog, among the SPIRE counterparts found through a MIPS prior position, the fraction of SPIRE sources having multiple IRAC potential associations is $57.6 \%$. SPIRE sources with MIPS counterparts are generally associated with high flux IRAC counterparts: only $4.0 \%$ of them have an IRAC flux below the $90 \%$ completeness limit (see Table 1). Moreover, multiple IRAC counterparts in a MIPS-IRAC search radius are very rare. This means that purely geometrical MIPS-IRAC associations are highly improbable. Therefore, we can safely assume that for almost all our SPIRE counterparts found through MIPS prior positions, there is a detected IRAC real counterpart. Given these assumptions and the results of our simulation, we expect that $49.7 \%$ of the SPIRE sources, aside from the real IRAC counterpart, have an additional purely geometrical association. Since we measure a real multiple association rate of $57.6 \%$, the additional $7.9 \%$ of these SPIRE sources must have real multiple IRAC components. Indeed, in our catalog, $\sim 6.1 \%$ of the SPIRE sample have multiple MIPS counterparts, and all of them have one IRAC counterpart inside the MIPS-IRAC search radius. The $\sim 2 \%$ difference confirms the reliability of the multiple SPIRE-MIPS associations discussed in Section 3.2.

As can be observed in the top panel of Figure 6, the $3.6 \mu \mathrm{m}$ flux for the SPIRE sources is generally lower when they are not detected at $24 \mu \mathrm{m}$. As explained in Section 3.2, the lower IRAC flux of these MIPS-undetected sources can be explained with the higher median redshift of the these sources. We find that $\sim 24 \%$ of this sample are below the $90 \%$ completeness level, resulting in an overall completeness of $\sim 85 \%$. Therefore, the probability of detecting the real counterpart inside the search radius is $P_{\mathrm{det}}^{\text {real }}=0.85$ while, from our simulation, the probability to find a purely geometrical IRAC counterpart is $P_{\mathrm{det}}^{\mathrm{geom}}=0.497$. We define the following products:

$$
\begin{gathered}
P_{1}=P_{\mathrm{det}}^{\mathrm{geom}}\left(1-P_{\mathrm{det}}^{\mathrm{real}}\right), \\
P_{2}=P_{\mathrm{det}}^{\mathrm{geom}} P_{\mathrm{det}}^{\mathrm{real}}, \\
P_{3}=\left(1-P_{\mathrm{det}}^{\mathrm{geom}}\right)\left(1-P_{\mathrm{det}}^{\mathrm{real}}\right), \\
P_{4}=\left(1-P_{\mathrm{det}}^{\mathrm{geem}}\right) P_{\mathrm{det}}^{\mathrm{real}} .
\end{gathered}
$$

Each SPIRE source can either have a possible counterpart (with probability $\left.P_{\mathrm{det}}=P_{1}+P_{2}+P_{4}\right)$ or not $\left(P_{\text {no- det }}=P_{3}\right)$, with $P_{1}+P_{2}+P_{3}+P_{4}=1$. All SPIRE sources in our catalog have one IRAC association. Assuming that the SPIRE position is always closer to the real IRAC counterpart than to the nearest geometrical association, their reliability can be estimated as:

$$
P_{\text {det }}^{\text {real }} \text { (cat) }=\frac{P_{2}+P_{4}}{P_{1}+P_{2}+P_{4}} \sim 0.92 .
$$

In reality, if a purely geometrical IRAC counterpart is present, it can be closer to the SPIRE position than the real counterpart. This is more probable when the IRAC-SPIRE distance is higher (and consequently the $P 3$ parameter lower). Moreover, as stated before, about $6 \%-8 \%$ of the SPIRE sources have more than a single real counterpart, further reducing the reliability for this sample. For all SPIRE sources in our catalog we report the number of potential IRAC counterparts in the search radius (N_IRAC_SPIRE) and the parameter $P 3$.

\subsection{Optical $R_{c}$ Band}

A central area of approximately one square degree was observed at the MPG/ESO $2.2 \mathrm{~m}$ telescope at La Silla with the Wide Field Imager (WFI) during 2010 October (P.I.: T. Takeuchi). The eight CCDs of the WFI camera cover a total area on the sky of $34^{\prime} \times 33^{\prime}$, with a pixel scale of 0 "' 24 . Four pointings with the $R_{c}$ broadband filter $\left(\lambda_{c}=6517.25 \AA\right)$ were obtained, covering a total area of $1.13 \mathrm{deg}^{2}$. Each pointing was observed with multiple exposures dithered to optimally remove the gaps between different CCDs and other CCD defects. The total exposure time varied between 2.2 and $1 \mathrm{hr}$.

The data were reduced with standard IRAF routines included in the NOAO mosaic software MSCRED. A Super-sky Flatfield correction was applied by dividing all of the science frames for the average of the non-aligned and source-subtracted science exposures. The final $R_{c}$ mosaic was created combining the images on the four pointings. In the final mosaic, the FWHM of the PSF was $1{ }^{\prime \prime} 0$.

Sources were extracted from the final mosaic using the SExtractor software. We considered only sources with five connected pixels above a threshold of $1.0 \sigma$ of the local background For each detected object, we recorded in the catalog the total AUTO flux. The photometric calibration of these data is obtained through the comparison with Bruzual \& Charlot (2003, BC03) template SEDs fitted to a large set of optical data. We used a set of measures obtained in the SIMES field for a selected sample of sources, in 13 different filters covering the spectral range between the $\mathrm{u}$ and the IRAC $4.5 \mu \mathrm{m}$ bands (I. Baronchelli et al. 2016, in preparation). Knowing the spectroscopic redshift of the selected sources (Sedgwick et al. 2011), we used a $\chi^{2}$ minimization technique (i.e., hyperzmass, Bolzonella et al. 2000) to find the best-fitting SED among the $\mathrm{BC} 03$ template SEDs. After comparing the extracted $R_{c}$ flux with the expected flux obtained from the convolution of the WFI- $R_{c}$ filter response with the best-fitting SED, we used the average difference to calibrate the extracted $R_{c}$ fluxes.

We computed the depth of the optical mosaic as follows. We measured the flux inside randomly distributed 1".9 diameter apertures and we fitted a Gaussian function to the symmetrized distribution of flux values. The $3 \sigma$ flux limit of the $R_{c}$ image is $0.53 \mu \mathrm{Jy}$.

In order to combine the optical and IR data, we searched the IRAC catalog for the closest counterpart to each $R$-detected source using a search radius of 0 !" 82 . The precise technique adopted is described in I. Baronchelli et al. (2016, in preparation). We found an optical counterpart for $\sim 55 \%$ of 


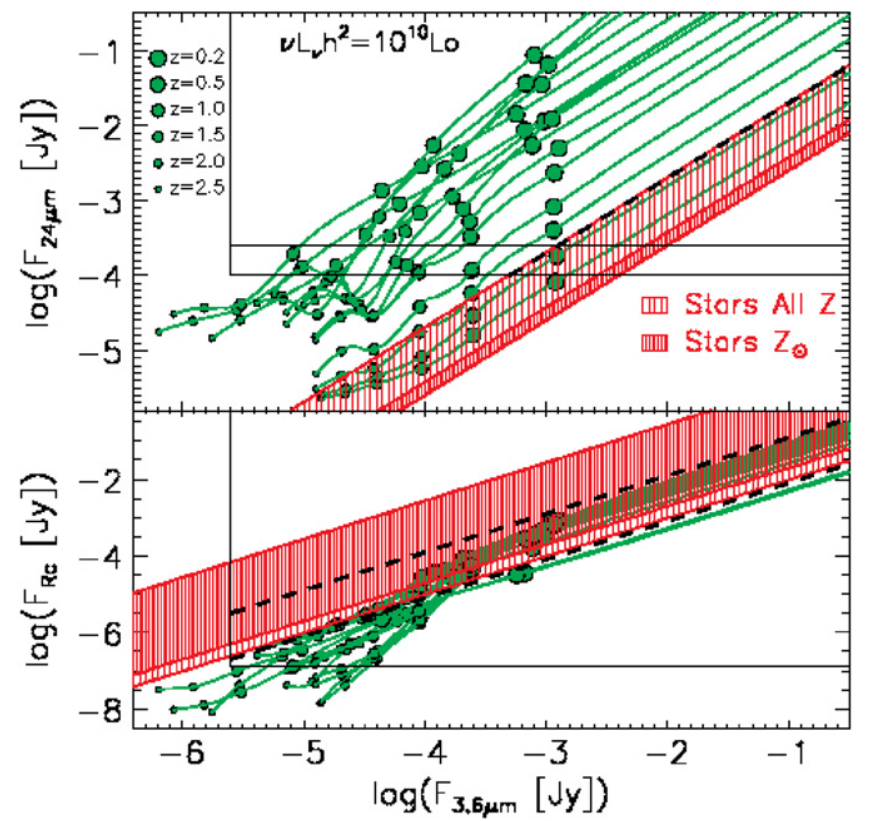

Figure 9. IRAC 3.6, MIPS $24 \mu \mathrm{m}$, and $R_{c}$ fluxes for a library of templates including both stars (red, using the stellar models of Kurucz 1993) and galaxies (green, in the range $0<z<2.5$ and normalized to $\nu L_{\nu} h^{2}=10{ }^{10} L_{\odot}$ at $3.6 \mu \mathrm{m}$ ). The selection thresholds used are represented with dashed black lines. The black boxes represent the areas covered by the plots of Figure 10 .

the IRAC-detected sources in our catalog which are covered in the $R_{c}$ band.

\subsection{Galaxy/Star Separation}

We perform the galaxy/star separation only in the central square degree area covered by the optical data. Our separation criteria, described in detail below, are based on a combination of diagnostics using optical, 3.6 and $24 \mu \mathrm{m}$ fluxes, as well as the surface brightness profile of each source from the optical data. Our selection criteria were calibrated using the stellar spectral models of Kurucz (1993) and a representative set of galaxy SED templates from Polletta et al. (2007), including ellipticals, spiral galaxies, starburst galaxies, and QSO templates.

We considered galaxy models in the range $0<z<2.5$ and with $\nu L_{\nu} h^{2}=10^{10} L_{\odot}$ at $3.6 \mu \mathrm{m}$, close to the characteristic $L^{*}$ luminosity (e.g., Babbedge et al. 2006; Franceschini et al. 2006). We used the Kurucz (1993) stellar models with abundances relative to solar ranging from $\log (Z)=1.0$ to $\log (Z)=-5.0$. In Figure 9, the differences between the areas occupied by the solar metallicity models and by the models with all the possible metallicities are shown. For each modeled star or galaxy, we computed the expected fluxes in the $3.6 \mu \mathrm{m}$, $24 \mu \mathrm{m}$, and $R_{c}$ bands. In Figure 9, galaxy tracks are shown in green (different sizes correspond to different redshifts). Regions in the diagnostic diagrams occupied by stars are shown as the shaded red band.

The bulk of the stellar emission for a galaxy is located at $\lambda$ $\sim 1.6 \mu \mathrm{m}$ (Simpson \& Eisenhardt 1999; Sawicki 2002). Stars are generally fainter at longer wavelengths, especially at the SPIRE wavelengths, where the galaxy spectra is dominated by the dust thermal emission. For this reason, among the SPIRE sources, the probability of detecting a star is negligible if compared to that of detecting a galaxy. Therefore, we classify

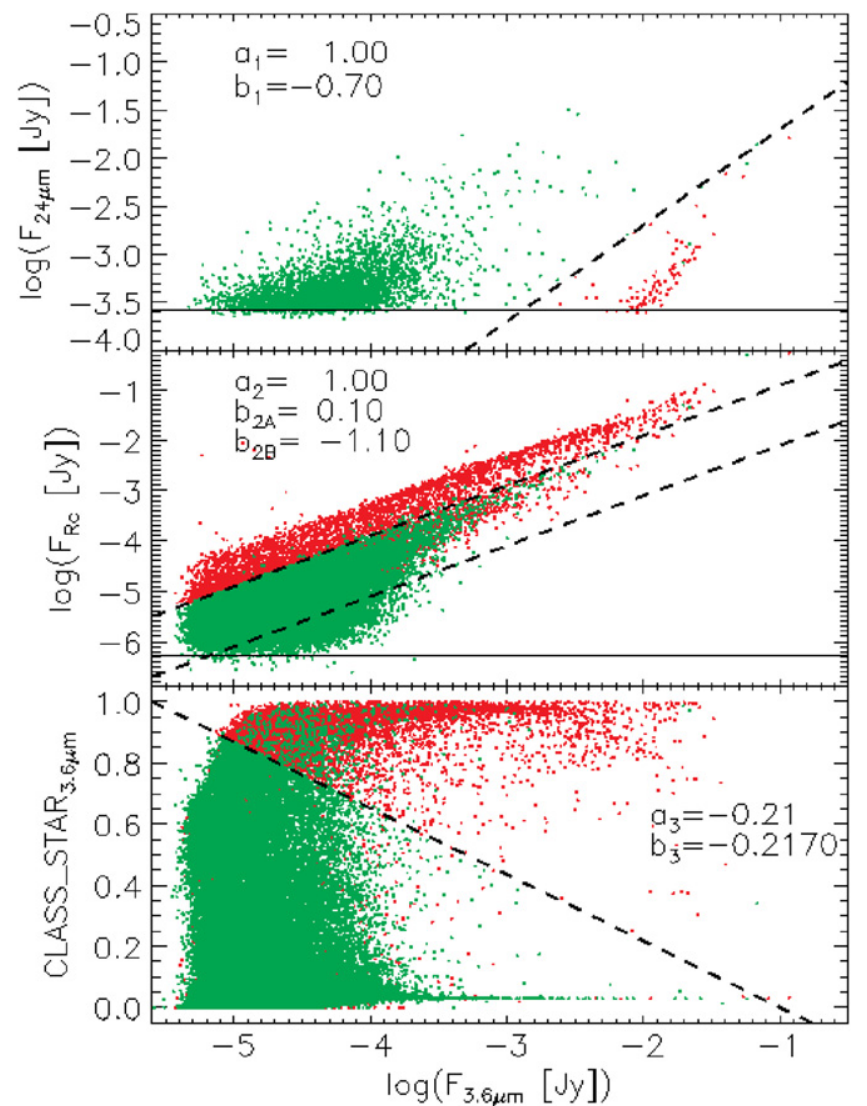

Figure 10. Galaxy/star separation. Same as Figure 9, but plotting the measurement for the objects detected in the SIMES field. In each panel, green points show sources that have been classified as galaxies by one of our criteria (see the text for details).

as a galaxy any object detected in one of the SPIRE bands. This assumption is confirmed by a visual inspection of the SPIRE sources on the IRAC images where the bright $3.6 \mu \mathrm{m}$ saturated stars are not SPIRE detected.

Then, following our diagnostics, we identify stars in the $3.6 \mu \mathrm{m}$ versus MIPS 24 plane (top panels of Figures 9 and 10). All sources with $\log \left(F_{24}[\mathrm{Jy}]\right)<\log \left(F_{3.6} \mu \mathrm{m}\right)-0.7$ are classified as stars. Because of the bright MIPS 24 flux limit $\left(\log F_{24}[\mathrm{Jy}] \sim-3.6\right)$, this selection misses faint IRAC-detected stars. We thus implement two additional constraints, based on the $R_{c}-3.6 \mu \mathrm{m}$ color (bottom panel of Figure 9 and middle panel of Figures 10) and the SExtractor CLASS_STAR ${ }^{26}$ parameter measured in the $3.6 \mu \mathrm{m}$ image (bottom panel in Figure 10). The SPIRE and $24 \mu \mathrm{m}$ undetected sources are classified as stars when $\log \left(F_{R_{c}}[\mathrm{Jy}]\right)>\log \left(F_{3.6 \mu \mathrm{m}}\right)+0.1$, and as galaxies when $\log \left(F_{R_{c}}[\mathrm{Jy}]\right)<\log \left(F_{3.6 \mu \mathrm{m}}\right)-1.1$. Between these two limits, or when no SPIRE, MIPS, or $R_{c}$ counterparts are found, we rely on the combination of the $3.6 \mu \mathrm{m}$ flux and the CLASS_STAR parameter to identify stars in our sample.

The reliability of the SExtractor CLASS_STAR parameter worsens at the faintest and brightest IRAC fluxes. At low fluxes (i.e., $F_{3.6 \mu \mathrm{m}} \lesssim 10^{-4} \mathrm{Jy}$ ), the shape of a galaxy looks similar to that of a point-like source, while the PSF wings of the brightest objects (i.e., $F_{3.6 \mu \mathrm{m}} \gtrsim 10^{-2.0} \mathrm{Jy}$ ) can be incorrectly interpreted as due to an extended

\footnotetext{
${ }^{26}$ CLASS_STAR $=0$ for galaxies, $=1$ for stars. This SExtractor output parameter quantifies the similarities between a source surface brightness profile and the profile of a point-like source.
} 


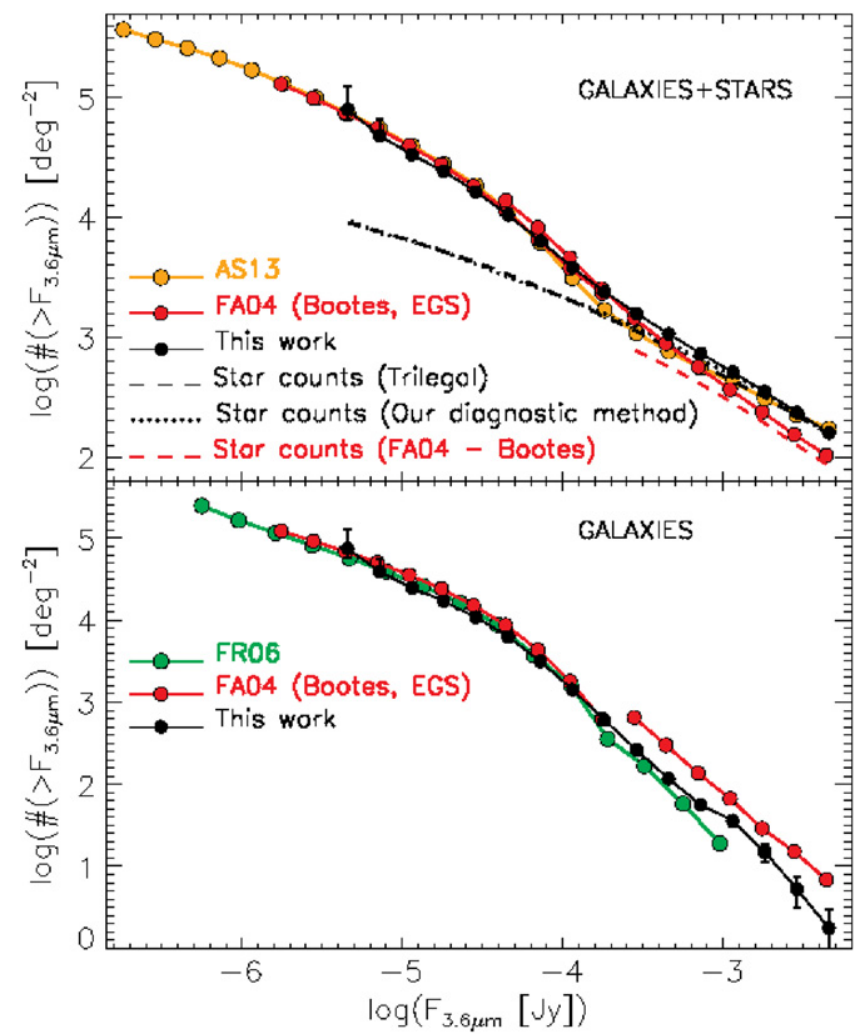

Figure 11. Top panel: Total (galaxy+stars) completeness-corrected integral number counts at $3.6 \mu \mathrm{m}$ from the SIMES survey (black symbols and line). Literature results are also shown in the figure as indicated by the legend, with AS13 = Ashby et al. (2013), FA04 = Fazio et al. (2004a), and FR06 = Franceschini et al. (2006). The observed and simulated (using TRILEGAL code) star counts computed in the SIMES field are represented by dotted and dashed black lines. FA04 star counts in the Boötes field are represented by a dashed red line. The FA04 counts are reported here for both the EGS (faint end) and the Boötes (bright end) field. For the Boötes field, source counts are provided fainter than stars and galaxies could be reliably separated using the method described in FA04. Above $F_{3.6 \mu \mathrm{m}} \sim 10^{-3.7} \mathrm{Jy}$, the total counts of AS13 are fully reproduced by stars alone.

profile by SExtractor. For these reasons, we introduce a flux dependent CLASS_STAR threshold: a source is identified as a star if CLASS_STAR $>-0.21 \log \left(F_{3.6 \mu \mathrm{m}}[\mathrm{Jy}]\right)-0.217$. We visually checked the correct identifications of all bright sources $\left(F_{3.6 \mu \mathrm{m}}>10^{-3.2} \mathrm{Jy}\right)$ using the $R_{c}$ image, correcting our counts for saturated stars wrongly identified as galaxies.

In order to assess the reliability of our diagnostic method, we compared our stellar number counts with those expected from a Milky Way model of stellar distribution. To compute the simulated stellar number counts, we used the population synthesis code TRILEGAL ${ }^{27}$ (Girardi et al. 2005) considering the position of the SIMES field. The result of this comparison is visible in Figure 11 where the simulated stellar number counts are represented by a dashed line while the counts of stars identified through our diagnostic method are reported using a dotted line. At all fluxes, we observe good agreement between observed and simulated stellar counts, confirming the reliability of our method.

\footnotetext{
${ }^{27}$ More information on this code can be found at: http://stev.oapd.inaf.it/cgibin/trilegal.
}

\section{RESULTS}

The IRAC-based multiwavelength photometric catalog together with the IRAC 3.6 and $4.5 \mu \mathrm{m}$ mosaics are released through the NASA/IPAC Infrared Science Archive (IRSA). In the following sections, we use this catalog to measure galaxy integral number counts (Section 4.1) and to search for $z \gtrsim 1.3$ galaxy clusters (Section 4.2). These are preliminary results, illustrative of those that will be allowed by the survey. We anticipate that future papers will improve the analysis once the deep optical Dark Energy Survey (Flaugher 2005) and CTIO-r (L. Barrufet et al. 2016, in preparation) data become available over the full survey area.

\subsection{Cumulative Number Counts}

We derive the galaxy-only and galaxy+stars (total) number counts from the $3.6 \mu \mathrm{m}$ map, and compare them with the results of Franceschini et al. (2006, FA04), Ashby et al. (2013, FR06), and Ashby et al. (2013, AS13). We computed the total counts in the entire SIMES field and the galaxies-only counts in the central square degree, where we use the optical $R_{c}$ band for the galaxy/star separation. All the number counts are corrected for incompleteness, with the values presented in Table 1 . We report the completeness-corrected SIMES $3.6 \mu \mathrm{m}$ integral number counts (both for galaxies+stars, and galaxies only), with the associated uncertainties, in Table 4.

The comparison between the cumulative SIMES number counts below $10^{-2.35} \mathrm{Jy}$ and those presented in Fazio et al. (2004a), Franceschini et al. (2006), and Ashby et al. (2013) are shown in Figure 11. Out of the three fields presented in FA04, we only compare their low- and intermediate-depth fields (more similar to the data presented here), i.e., $\sim 3 \times 3$ area in the the Boötes field and $0.17 \times 2.0$ area in the EGS field, respectively. The FA04 galaxy and total number counts in the EGS field are presented in differential form, starting at the highest flux of $10^{-3.76} \mathrm{Jy}$. To convert them from differential to cumulative, we use our cumulative galaxy number counts at $10^{-3.76} \mathrm{Jy}$ as a starting point. For the FA04 counts in the Boötes field, we do not use any starting point because they are reported through high fluxes (i.e., $10^{-1.76} \mathrm{Jy}$ ) where counts assume fractional values. FR06 presents cumulative number counts for galaxy only and no starting point is needed. On the other hand, AS13 presents total differential counts. As for the FA04 EGS counts, we used the SIMES cumulative counts at the flux of the brightest AS13 bin as staring point.

At $3.6 \mu \mathrm{m}$ fluxes fainter than $\sim 10^{-4} \mathrm{Jy}$, our cumulative galaxy number counts, shown in the bottom panel of Figure 11, agree well with the three reference surveys. Above $\sim 10^{-4} \mathrm{Jy}$, differences are observed among all the works. Our galaxy counts fall in between those of FA04 for the Boötes field and the FR06 counts. The scatter at bright fluxes is likely ascribable to two main reasons. First, the field to field variation combined with the bias against bright sources affecting deep field small areas. The deep GOODS-south area (Dickinson et al. 2003) analyzed in FR06, among other reasons, was selected for being far from bright sources. A second likely explanation is the uncertainty in the galaxy/star separation. In particular, we note that the difference between our galaxy counts in the brightest bin and the FA04 counts at the same flux level can be fully explained with a $<5 \%$ uncertainty in our corresponding stellar counts. 
Table 4

Raw and Completeness Corrected Integral Number Counts at $3.6 \mu \mathrm{m}$

\begin{tabular}{|c|c|c|c|c|c|c|c|c|c|}
\hline \multirow{2}{*}{$\begin{array}{l}\text { Flux } \\
(\log (J y))\end{array}$} & \multicolumn{2}{|c|}{ Raw Counts $\left(>S_{3.6}\right)$} & \multirow{2}{*}{$\begin{array}{l}\text { Corr. } \\
\text { Fact. }\end{array}$} & \multicolumn{6}{|c|}{ Corrected Counts $\left(>S_{3.6}\right)$} \\
\hline & $\begin{array}{c}N_{\mathrm{GAL}} \\
\left(\mathrm{deg}^{-2}\right)\end{array}$ & $\begin{array}{c}N_{\mathrm{TOT}} \\
\left(\operatorname{deg}^{-2}\right)\end{array}$ & & $\begin{array}{c}N_{\mathrm{GAL}} \\
\left(\mathrm{deg}^{-2}\right)\end{array}$ & $\begin{array}{c}N_{\mathrm{GAL}}^{\mathrm{Inf}} \\
\left(\mathrm{deg}^{-2}\right)\end{array}$ & $\begin{array}{c}\mathrm{N}_{\mathrm{GAL}}^{\mathrm{Sup}} \\
\left(\mathrm{deg}^{-2}\right)\end{array}$ & $\begin{array}{c}N_{\mathrm{TOT}} \\
\left(\mathrm{deg}^{-2}\right)\end{array}$ & $\begin{array}{c}N_{\mathrm{TOT}}^{\mathrm{Inf}} \\
\left(\mathrm{deg}^{-2}\right)\end{array}$ & $\begin{array}{c}N_{\mathrm{TOT}}^{\text {Sup }} \\
\left(\mathrm{deg}^{-2}\right)\end{array}$ \\
\hline-2.34 & $2 \pm 1$ & $159 \pm 13$ & 1.0 & 2 & 1 & 3 & 159 & 154 & 163 \\
\hline-2.54 & $5 \pm 2$ & $236 \pm 15$ & 1.0 & 5 & 3 & 7 & 236 & 230 & 241 \\
\hline-2.74 & $15 \pm 4$ & $352 \pm 19$ & 1.0 & 15 & 11 & 19 & 352 & 346 & 359 \\
\hline-2.94 & $36 \pm 6$ & $511 \pm 23$ & 1.0 & 36 & 30 & 42 & 511 & 503 & 519 \\
\hline-3.14 & $56 \pm 8$ & $735 \pm 27$ & 1.0 & 56 & 49 & 63 & 735 & 725 & 745 \\
\hline-3.34 & $117 \pm 11$ & $1069 \pm 33$ & 1.0 & 117 & 107 & 127 & 1069 & 1057 & 1081 \\
\hline-3.54 & $263 \pm 16$ & $1583 \pm 40$ & 1.0 & 263 & 247 & 278 & 1583 & 1568 & 1597 \\
\hline-3.74 & $604 \pm 25$ & $2388 \pm 49$ & 0.997 & 603 & 580 & 627 & 2395 & 2377 & 2414 \\
\hline-3.94 & $1416 \pm 38$ & $3803 \pm 62$ & 0.990 & 1421 & 1385 & 1457 & 3841 & 3817 & 3865 \\
\hline-4.14 & $3116 \pm 56$ & $6250 \pm 79$ & 0.981 & 3150 & 3096 & 3204 & 6373 & 6341 & 6405 \\
\hline-4.34 & $6151 \pm 78$ & $10187 \pm 101$ & 0.972 & 6262 & 6188 & 6338 & 10481 & 10443 & 10522 \\
\hline-4.54 & $10646 \pm 103$ & $15832 \pm 126$ & 0.963 & 10904 & 10802 & 11010 & 16434 & 16376 & 16503 \\
\hline-4.74 & $16629 \pm 129$ & $23149 \pm 152$ & 0.954 & 17143 & 17012 & 17292 & 24274 & 24194 & 24391 \\
\hline-4.94 & $23892 \pm 155$ & $31579 \pm 178$ & 0.941 & 24859 & 24679 & 25968 & 33543 & 33407 & 34820 \\
\hline-5.14 & $32069 \pm 179$ & $39748 \pm 199$ & 0.827 & 38908 & 34570 & 56867 & 48091 & 43760 & 66032 \\
\hline-5.34 & $36886 \pm 192$ & $43923 \pm 210$ & 0.549 & 74959 & 57300 & 126954 & 79997 & 64693 & 125058 \\
\hline
\end{tabular}

Note.

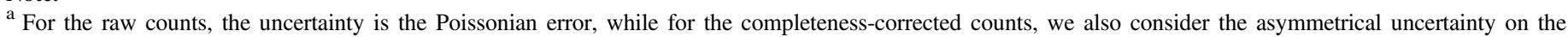
estimated completeness curve.

The star counts in the SIMES area agree well with the counts simulated using the TRILEGAL software (Girardi et al. 2005). For the TRILEGAL simulation, based on a statistical description of the stellar distribution in the Milky Way, we set the same coordinates of the SIMES field in order to obtain comparable results. Instead, the FA04 stellar counts refer to a different area of the sky (Boötes), where stellar counts are expected to be lower, because of the higher galactic latitude. This is indeed observed in FA04 (upper panel of Figure 11) and it is confirmed by the results of a second TRILEGAL simulation that we performed in an area centered in the (Boötes) region. In the brightest flux bin, simulated stellar counts resulted $\sim 20 \%$ lower than FA04 real counts. This indicates that the different galaxy counts in the SIMES and Boötes fields are unlikely due to an underestimation of FA04 stellar counts. Similar differences in stellar counts, when comparing different areas of the sky, can be observed e.g., in Papovich C. et al. (2015).

\subsection{Selection of Clusters at Intermediate Redshifts}

Galaxy clusters - through their space density and evolution with cosmic time - provide crucial information on the physical processes involved in cosmic structure formation. Representing the most extreme density environments, they provide galaxy samples with near coeval formation histories, and are thus ideal laboratories in which to investigate the interplay between galaxy evolution and environment, including the relative importance of triggering/quenching of SF and AGN activity on galaxy assembly. Papovich et al. (2010) extended the search for galaxy clusters to $z>1.5$ by selecting galaxy cluster candidates from the SWIRE survey solely as over-densities of galaxies with red IRAC colors, satisfying [3.6]-[4.5] $>-0.2 \mathrm{mag}$ (see also Rettura et al. 2014, for a recent application of this technique). The idea behind the method is simple, and is based on the $1.6 \mu \mathrm{m}$ stellar peak progressively moving out of the $3.6 \mu \mathrm{m}$ and entering the $4.5 \mu \mathrm{m}$ filter as redshift increases above $z \sim 0.7$.
In order to identify over-densities of red galaxies, we proceeded in a manner similar to Rettura et al. (2014). Briefly, before searching for spatial over-densities, we preselected only those galaxies which satisfied the following conditions: IRAC $[3.6]-[4.5]>-0.2,19.5<[4.5]<21.5$, and $\mathrm{S} / \mathrm{N}>3$ and 5 , at 3.6 and $4.5 \mu \mathrm{m}$, respectively. Similar cuts have been effectively used by various programs (Galametz et al. 2010, 2013; Papovich et al. 2010; Gettings et al. 2012; Muzzin et al. 2013; Rettura et al. 2014). In the central square degree where our WFI- $R_{c}$ data are available, we also require selected galaxies to have $F_{R_{c}}<14.5 \mu \mathrm{Jy}$ (this condition helps to broadly reject contaminants at $z<0.3$ ). For each galaxy $j$ in the selected sample, we then computed the quantity $\Delta N_{C C}=N_{1^{\prime}}-N_{5^{\prime}-8^{\prime}}$, i.e., the difference between the number of red galaxies within $1 ! 0$ from the $j$ th galaxy $\left(N_{1}\right)$ and the number of red galaxies in the background, which we computed inside an annulus of radius $5 ! 0-8 ! 0$, normalized to a circular area of $1 ! 0$ radius $\left(N_{5^{\prime}-8^{\prime}}\right)$. All of the counts are corrected for incompleteness using the results of the simulation discussed in Section 2.3, and computing the number of galaxies within a given distance from galaxy $j$, as $N_{j}=\sum_{i}^{N} \frac{1}{C_{i}(3.6)}$, where the sum is over all $N$ galaxies within 1!.0 from galaxy $j$, and $C_{i}$ is the completeness corresponding to the $3.6 \mu \mathrm{m}$ flux of galaxy $i$.

The observed distribution of the excess number of objects (within 1!.0) with respect to the local background $\left(\Delta N_{C C}\right)$ is shown in Figure 12 (top panel), together with the best-fit Gaussian distribution computed using values of $\Delta N_{C C}<3$. The best-fit Gaussian distribution has a mean of 0.45 and a standard deviation of $\sigma=4.6$, consistent with the best-fit values obtained by Rettura et al. (2014) on similar depth data, on more than ten times the area. The Gaussian function can be used to describe the probability of observing a given excess number of objects around a galaxy, under the null hypothesis $(\mathrm{H})$ that the galaxy does not belong to a cluster. In order to identify only those galaxies located within clusters we proceed following the Benjamini-Hochberg procedure (BH; Benjamini $\&$ Hochberg 1995), which minimizes the false discovery rate at 
Table 5

Multiwavelength Catalog Columns and Description ${ }^{\mathrm{a}}$

\begin{tabular}{|c|c|c|}
\hline Column & Example & Content \\
\hline ID & 163210 & Identification number for IRAC 1 detected sources. \\
\hline R.A._I1 & 71.040682 & IRAC $3.6 \mu \mathrm{m}$ R.A. coordinate \\
\hline decl._I1 & -53.615640 & IRAC $3.6 \mu \mathrm{m}$ decl. coordinate \\
\hline FLUX_I1 & 4.58311 & IRAC $3.6 \mu \mathrm{m}$ total mJy flux (FLUX_AUTO) \\
\hline FLUXERR_I1 & 0.00820884 & IRAC $3.6 \mu \mathrm{m}$ mJy flux associated uncertainty \\
\hline N_SIGMA & 813.635 & IRAC $3.6 \mu \mathrm{m}$ Signal to noise ratio connected to average coverage \\
\hline FLUX_I2 & 3.05153 & IRAC $4.5 \mu \mathrm{m}$ total mJy flux (FLUX_AUTO, I1 prior position used) \\
\hline FLUXERR_I2 & 0.00781307 & IRAC $4.5 \mu \mathrm{m}$ mJy flux associated uncertainty \\
\hline R.A._24 & 71.040554 & $\begin{array}{l}\text { R.A. coordinate for MIPS } 24 \mu \mathrm{m} \text { sources corrected for } \\
\text { systematic shift }\left(-0{ }^{\prime \prime} 165\right)\end{array}$ \\
\hline decl._24 & -53.615536 & $\begin{array}{c}\text { decl. coordinate for MIPS } 24 \mu \mathrm{m} \text { sources corrected for } \\
\text { systematic shift }\left(-0.0^{\prime \prime} 489\right)\end{array}$ \\
\hline O_R.A._24 & 71.040600 & original RA coordinate for MIPS 24 sources $^{\mathrm{b}}$ \\
\hline O_decl._24 & -53.615400 & original decl. coordinate for MIPS 24 sources $^{\mathrm{b}}$ \\
\hline FLUX_24 & 3.98400 & MIPS $24 \mu \mathrm{m}$ mJy flux ${ }^{\mathrm{b}}$ \\
\hline FLUXERR_24 & 0.0600000 & MIPS $24 \mu \mathrm{m}$ mJy flux uncertainty ${ }^{\mathrm{b}}$ \\
\hline FLUX_70 & 20.0000 & MIPS $70 \mu \mathrm{m}$ mJy flux ${ }^{\mathrm{b}}$ \\
\hline FLUXERR_70 & 3.63636 & MIPS $70 \mu \mathrm{m}$ mJy flux uncertainty ${ }^{\mathrm{b}}$ \\
\hline R.A._SPIRE & 71.039836 & $\begin{array}{l}\text { R.A. coordinate for SPIRE sources corrected for } \\
\text { systematic shift }\left(-0{ }^{\prime \prime} 199\right)\end{array}$ \\
\hline decl._SPIRE & -53.615458 & $\begin{array}{l}\text { decl. coordinate for SPIRE sources corrected for } \\
\text { systematic shift }(-0,443)\end{array}$ \\
\hline O_R.A._SPIRE & 71.039856 & original R.A. SPIRE coordinate ${ }^{c}$ \\
\hline O_decl._SPIRE & -53.615330 & original decl. SPIRE coordinate $^{c}$ \\
\hline FLUX_250 & 92.635274 & SPIRE $250 \mu$ m mJy flux ${ }^{c}$ \\
\hline FLUXERR_250 & 2.3467732 & SPIRE 250 mJy flux uncertainty ${ }^{c}$ \\
\hline FLUX_350 & 38.933088 & SPIRE $350 \mu \mathrm{m} \mathrm{mJy} \mathrm{flux}{ }^{\mathrm{c}}$ \\
\hline FLUXERR_350 & 4.4716398 & SPIRE 350 mJy flux uncertainty ${ }^{c}$ \\
\hline FLUX_500 & 20.702205 & SPIRE $500 \mu$ m flux $^{\mathrm{c}}$ \\
\hline FLUXERR_500 & 4.0425355 & SPIRE 500 mJy flux uncertainty ${ }^{c}$ \\
\hline R.A._OPT & -53.615583 & R.A. coordinate for WFI- $R_{c}$ \\
\hline decl._OPT & -53.615583 & decl. coordinate for WFI- $R_{c}$ \\
\hline FLUX_R_WFI & 3.87101 & WFI- $R_{c}$ total mJy flux (FLUX_AUTO) \\
\hline FLUXERR_R_WFI & 0.00968389 & WFI- $R_{c}$ total mJy flux uncertainty \\
\hline $\mathrm{P} 1$ & 0.84638566 & $\begin{array}{l}\text { SPIRE-MIPS reliability indicator } \\
\text { (ranges from } 0 \text { to } 1 \text {, where } 0=\text { bad, } 1=\text { good) }\end{array}$ \\
\hline N_MIPS_SPIRE & 1 & Number of MIPS counterparts for the SPIRE source \\
\hline $\mathrm{P} 2$ & 0.85814963 & $\begin{array}{l}\text { MIPS-IRAC reliability indicator } \\
\text { (ranges from } 0 \text { to } 1 \text {, where } 0=\text { bad, } 1=\text { good) }\end{array}$ \\
\hline N_IRAC_MIPS & 1 & Number of IRAC counterparts for the MIPS source \\
\hline $\mathrm{P} 3$ & 0.80220842 & $\begin{array}{l}\text { SPIRE-IRAC reliability indicator } \\
\text { (ranges from } 0 \text { to } 1 \text {, where } 0=\text { bad, } 1=\text { good) }\end{array}$ \\
\hline N_IRAC_SPIRE & 1 & Number of IRAC counterparts for the SPIRE source \\
\hline CLASS_STAR_I1 & 0.0286267 & $\begin{array}{l}\text { SExtractor CLASS_STAR parameter for IRAC } 3.6 \mu \mathrm{m} \\
\quad \text { (ranges from } 0 \text { to } 1 \text {, where } 0=\text { galaxy, } 1=\text { star) }\end{array}$ \\
\hline A_I1 & 6.28684 & Semimajor axis in arcseconds \\
\hline B_I1 & 3.39138 & Semiminor axis in arcseconds \\
\hline SIGMA & 0.00145476 & IRAC $3.6 \mu \mathrm{m}$ sky sigma value (depends on the coverage) \\
\hline COVERAGE & 9.66118 & $\begin{array}{l}\text { Average coverage computed over an area of } 49 \text { pixels } \\
\text { centered on the } 3.6 \mu \mathrm{m} \text { coordinates }\end{array}$ \\
\hline AP1_FLUX_I1 & 0.00118364 & IRAC $3.6 \mu \mathrm{m}$ mJy aperture flux ( 4 !" 8 ap. diameter). \\
\hline AP1_FLUXERR_I1 & 0.00118364 & IRAC $3.6 \mu \mathrm{m}$ mJy aperture flux uncertainty (4!" 8 ap. diameter). \\
\hline AP2_FLUX_I1 & 0.00184539 & IRAC $3.6 \mu \mathrm{m}$ mJy aperture flux (7!' 2 ap. diameter). \\
\hline AP2_FLUXERR_I1 & 0.00184539 & IRAC $3.6 \mu \mathrm{m}$ mJy aperture flux uncertainty ( 7 !! 2 ap. diameter). \\
\hline AP3_FLUX_I1 & 0.00291065 & IRAC $3.6 \mu \mathrm{m}$ mJy aperture flux ( 12 ". 0 ap. diameter). \\
\hline AP3_FLUXERR_I1 & 0.00291065 & IRAC $3.6 \mu \mathrm{m}$ mJy aperture flux uncertainty ( $12{ }^{\prime \prime} 0$ ap. diameter). \\
\hline AP1_FLUX_I2 & 0.00118364 & IRAC $4.5 \mu \mathrm{m}$ mJy aperture flux (4". 8 ap. diameter). \\
\hline AP1_FLUXERR_I2 & 0.00118364 & IRAC $4.5 \mu \mathrm{m}$ mJy aperture flux uncertainty ( 4 ". 8 ap. diameter). \\
\hline AP2_FLUX_I2 & 0.00184539 & IRAC $4.5 \mu \mathrm{m}$ mJy aperture flux (7!' 2 ap. diameter). \\
\hline AP2_FLUXERR_I2 & 0.00184539 & IRAC $4.5 \mu \mathrm{m}$ mJy aperture flux uncertainty (7!' 2 ap. diameter). \\
\hline AP3_FLUX_I2 & 0.00291065 & IRAC $4.5 \mu \mathrm{m}$ mJy aperture flux (12". 0 ap. diameter). \\
\hline AP3_FLUXERR_I2 & 0.00291065 & IRAC $4.5 \mu \mathrm{m}$ mJy aperture flux uncertainty ( $12{ }^{\prime \prime} 0$ ap. diameter). \\
\hline
\end{tabular}

Notes.

a All the fluxes are expressed in mJy and the coordinates are in degrees. All the fluxes are "total" and do not need any further aperture correction, unless differently specified. The IRAC aperture fluxes reported here for the $4 . " 8,7 ! .2$, and $12 . " 0$ diameter apertures are not aperture corrected; to obtain the correspondent total flux, the IRAC handbook aperture corrections are needed. The counterpart distances are expressed in arcseconds. The catalog is released through the NASA/IPAC Infrared Science Archive (IRSA) service.

${ }^{\mathrm{b}}$ From Clements et al. (2011).

${ }^{\mathrm{c}}$ From SPIRE XID catalogs (DR2, Roseboom et al. 2010; Smith et al. 2012; Wang et al. 2014). 

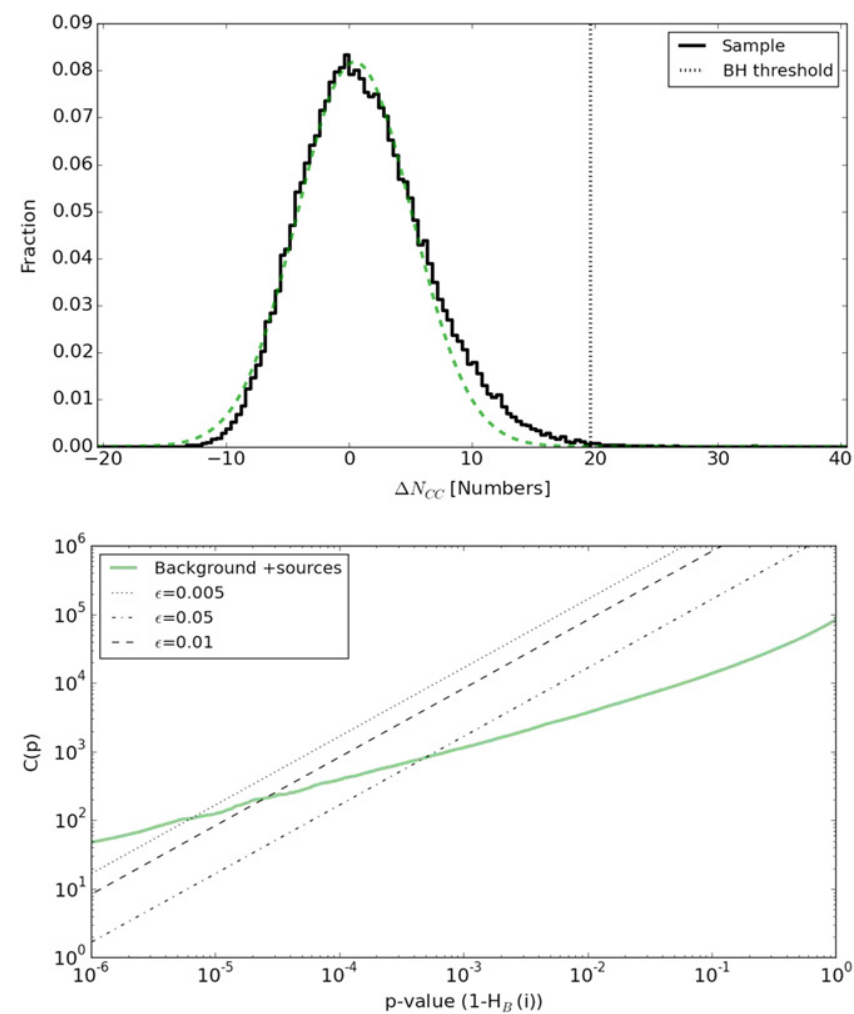

Figure 12. Top panel: distribution of the excess number of objects (within 1!.0) with respect to the local background for all galaxy candidates at $z>1.3$. The green dashed line shows the best-fit Gaussian distribution fitted for values of $\Delta N_{C C}<7$, and the probability of observing a given excess number of objects around a galaxy, under the null hypothesis that the galaxy does not belong to a cluster. Bottom panel: illustration of the Benjamini-Hochberg procedure: the green solid line shows the cumulative distribution of galaxies' $p$-values computed under the assumption of the null hypothesis (i.e., using the Gaussian best-fit parameters). The black lines correspond to different levels of contamination of the final sample.

a level $\epsilon$. Briefly, for each galaxy we compute its $p$-value under the null hypothesis $\mathrm{H}$. The $p$-values are ordered in increasing order and denoted by $p_{1}, \ldots, p_{N}$. The cumulative distribution of the $p$-values is shown in Figure 12, bottom panel. Note that the value of $C(p)$ corresponds to the index $j$ of each galaxy (the galaxies were sorted according to their $p$-value). For a given $\epsilon$, we compute the critical $p$-value by finding the largest $j$ such that $p_{j} \leqslant \frac{k}{N} \epsilon$. The corresponding $\Delta N_{C C}$ is then the cutoff value we use to identify galaxies belonging to a cluster. The $\mathrm{BH}$ procedure ensures that the false discovery rate is smaller than $(\epsilon \times 100) \%$. In Figure 12 we show the curves corresponding to various values of $\epsilon$. For the cluster selection we used the conservative value $\epsilon=0.005$, which corresponds to objects with $\Delta N_{C C} \geqslant 19.7$ (indicated by the vertical dotted line in the top panel of Figure 12).

The procedure above identifies galaxies residing in over dense regions, and thus can identify multiple galaxies belonging to the same over density. We follow Papovich (2008) and Rettura et al. (2014) and merge the cluster candidates by applying a friends-of-friends algorithm with a linking length of $1 ! 5$, corresponding to approximately $0.8 \mathrm{Mpc}$ at $z=1.5$. With this algorithm, we identify 27 unique galaxy clusters. An example of a detected cluster in the central region where optical data are available is shown in Figure 13. The density of clusters in the SIMES area $\left(3.8 \pm 0.7\right.$ clusters $\left.\mathrm{deg}^{-2}\right)$

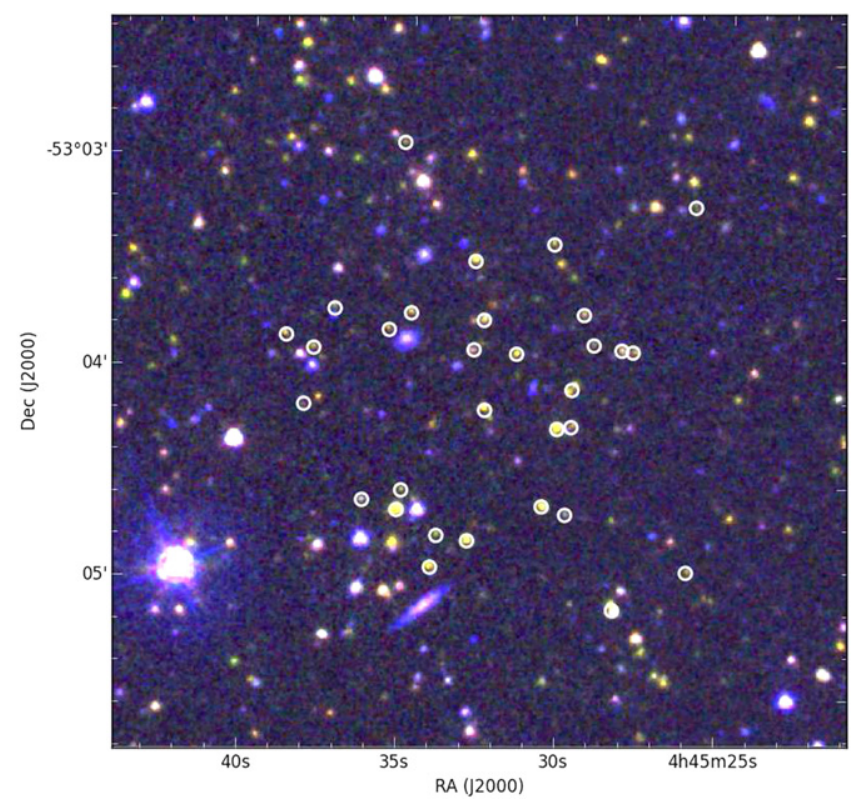

Figure 13. False color image (red $=4.5 \mu \mathrm{m}$, green $=3.6 \mu \mathrm{m}$, and blue $=R_{c}$ ) showing a $3.7 \times 3 ! 7$ region around one of the galaxy cluster identified in the central square degree where optical data are available. Open circles show galaxies with red 3.6-4.5 $\mu \mathrm{m}$ color, $3.6 \mu \mathrm{m}$ and $R_{c}$ magnitudes fainter than 18.7 and 21 , respectively.

is consistent with the density found by Rettura et al. (2014; $3.0 \pm 0.2$ clusters $\operatorname{deg}^{-2}$ ).

\section{SUMMARY}

We presented SIMES in the SEP field and the multiwavelength catalog of sources based on the $3.6 \mu \mathrm{m}$ detections. The survey covers an area of $7.74 \mathrm{deg}^{2}$ to a depth of $\sim 5.80 \mu \mathrm{Jy}$ $(3 \sigma)$ at $3.6 \mu \mathrm{m}$ and $5.25 \mu \mathrm{Jy}$ at $4.5 \mu \mathrm{m}$. We estimate $90 \%$ and $50 \%$ completeness levels in the $3.6 \mu \mathrm{m}$ band at 14 and $9 \mu \mathrm{Jy}$, respectively. Table 5 describes all the columns in our photometric catalog.

The SIMES region has been targeted by numerous multiwavelengths surveys spanning the UV to the far-IR and radio regimes. The addition of the Spitzer-IRAC observations is crucial for computing reliable photometric redshifts and stellar masses for all galaxies detected in this region by the Herschel satellite. The IRAC observation presented here allowed us to identify the optical/IR counterparts of the starforming galaxies and AGNs detected at the far-IR wavelengths. We included in our multiwavelength catalog the WFI- $R_{c}$, MIPS $24 \mu \mathrm{m}$, SPIRE 250, 350 , and $500 \mu \mathrm{m}$ fluxes of the counterparts that we identified by searching for the closest neighbor. The reliability (i.e., fraction of spurious detections introduced) of these associations is quantified through the indicator " $P$ " that we computed for each MIPS and SPIRE detected source. The possibility of a direct IRAC-SPIRE association is also discussed. The full catalog is available through the NASA/IPAC IRSA.

We reported $3.6 \mu \mathrm{m}$ galaxy and total (galaxy and stars) number counts in the SIMES field and compared them with literature results obtained in different fields. Below $F_{3.6 \mu \mathrm{m}}=10^{-4.0} \mathrm{Jy}$ our galaxy counts are more in agreement with Franceschini et al. (2006) than with Fazio et al. (2004a). Above $F_{3.6 \mu \mathrm{m}}=10^{-4.0} \mathrm{Jy}$ galaxy counts in the SIMES field are in between those of Fazio et al. (2004a) and Franceschini et al. (2006). While our galaxy number counts are computed within 
the area with optical imaging, our total counts are calculated in the whole SIMES area.

Finally, using the method proposed in Papovich et al. (2010), we identified 27 galaxy clusters at $z>1.3$. Although preliminary (only part of the field at this point is covered by optical data), the surface density of the galaxy clusters in SIMES is consistent with that reported in Rettura et al. (2014).

Further deep observations in optical bands will soon be available (I. Baronchelli et al. 2016, in preparation). Such observations will allow us to improve our estimates and the measure of precise photometric redshifts for the galaxies in the SIMES field. The correlation among the near- and far-IR bands will be further improved using the available $90 \mu \mathrm{m}$ Akari data presented in Małek et al. (2014). These data will also allow for an extensive study of the dust thermal emission in these spectral regions.

We thank the referee for the useful comments that improved the presentation of the paper. I.B. and G.R. acknowledge support from ASI (Herschel Science Contract 2011aI/005/ 011/0). C.S. and I.B. acknowledge support from NASA JPL/ Spitzer grant RSA 1449911, provided for the SIMES project. S.M. acknowledges financial support from the Institut Universitaire de France (IUF), of which she is senior member. M.V. acknowledges support from the European Commission Research Executive Agency (FP7-SPACE-2013-1 GA 607254) and the Italian Ministry for Foreign Affairs and International Cooperation (PGR GA ZA14GR02). This research has made use of data from the HerMES project (http://hermes. sussex.ac.uk/). HerMES is a Herschel Key Program using Guaranteed Time from the SPIRE instrument team, ESAC scientists, and a mission scientist. The HerMES data were accessed through the Herschel Database in Marseille (HeDaM -http://hedam.lam.fr), operated by CeSAM and hosted by the Laboratoire d'Astrophysique de Marseille. This research has made use of the NASA/IPAC Infrared Science Archive, which is operated by the Jet Propulsion Laboratory, California Institute of Technology, under contract with the National Aeronautics and Space Administration. M.V. acknowledges support from the European Commission Research Executive Agency (FP7-SPACE-2013-1 GA 607254) and the Italian Ministry for Foreign Affairs and International Cooperation (PGR GA ZA14GR02).

\section{REFERENCES}

Ashby, M. L. N., Willner, S. P., Fazio, G. G., et al. 2013, ApJ, 769, 80

Babbedge, T. S. R., Rowan-Robinson, M., Vaccari, M., et al. 2006, MNRAS, 370, 1159

Bedregal, A. G., Scarlata, C., Henry, A. L., et al. 2013, ApJ, 778, 126

Benjamini, Y., \& Hochberg, Y. 1995, Journal of the Royal Statistical Society Series B, 57, 289

Bertin, E., \& Arnouts, S. 1996, A\&AS, 117, 393

Bolzonella, M., Miralles, J., \& Pelló, R. 2000, A\&A, 363, 476
Bruzual, G., \& Charlot, S. 2003, MNRAS, 344, 1000

Capak, P. L., Teplitz, H. I., Brooke, T. Y., Laher, R., \& Science Center, S. 2013, in American Astronomical Society Meeting Abstracts, \#221, 340.06 Chary, R., \& Elbaz, D. 2001, ApJ, 556, 562

Clements, D. L., Bendo, G., Pearson, C., et al. 2011, MNRAS, 411, 373

Daddi, E., Dickinson, M., Morrison, G., et al. 2007, ApJ, 670, 156

Dale, D. A., Gil de Paz, A., Gordon, K. D., et al. 2007, ApJ, 655, 863

Dickinson, M., Giavalisco, M., \& GOODS Team 2003, The Mass of Galaxies at Low and High Redshift, ed. R. Bender, \& A. Renzini (Berlin: SpringerVerlag), 324

Elbaz, D., Dickinson, M., Hwang, H. S., et al. 2011, A\&A, 533, A119

Fazio, G. G., Ashby, M. L. N., Barmby, P., et al. 2004a, ApJS, 154, 39

Fazio, G. G., Hora, J. L., Allen, L. E., et al. 2004b, ApJS, 154, 10

Flaugher, B. 2005, IJMPA, 20, 3121

Franceschini, A., Rodighiero, G., Cassata, P., et al. 2006, A\&A, 453, 397

Galametz, A., Stern, D., Pentericci, L., et al. 2013, A\&A, 559, A2

Galametz, A., Vernet, J., De Breuck, C., et al. 2010, A\&A, 522, A58

Gettings, D. P., Gonzalez, A. H., Stanford, S. A., et al. 2012, ApJL, 759, L23

Girardi, L., Groenewegen, M. A. T., Hatziminaoglou, E., \& da Costa, L. 2005, A\&A, 436, 895

Graham, A. W., \& Driver, S. P. 2005, PASP, 22, 118

Griffin, M. J., Abergel, A., Abreu, A., et al. 2010, A\&A, 518, L3

Gruppioni, C., Pozzi, F., Rodighiero, G., et al. 2013, MNRAS, 432, 23

Kirkpatrick, A., Pope, A., Alexander, D. M., et al. 2012, ApJ, 759, 139

Kron, R. G. 1980, ApJS, 43, 305

Kurucz, R. 1993, ATLAS9 Stellar Atmosphere Programs and $2 \mathrm{~km} / \mathrm{s}$ grid. Kurucz CD-ROM No. 13 (Cambridge, MA: Smithsonian Astrophysical Observatory)

Magnelli, B., Lutz, D., Santini, P., et al. 2012, A\&A, 539, A155

Makovoz, D., \& Khan, I. 2005, in ASP Conf. Ser. 347, Astronomical Data Analysis Software and Systems XIV, ed. P. Shopbell, M. Britton, \& R. Ebert (San Francisco, CA: ASP), 81

Makovoz, D., Khan, I., \& Moshir, M. 2005, PASP, 117, 274

Małek, K., Pollo, A., Takeuchi, T. T., et al. 2014, A\&A, 562, A15

Matsuhara, H., Wada, T., Matsuura, S., et al. 2006, PASJ, 58, 673

Matsuura, S., Shirahata, M., Kawada, M., et al. 2011, ApJ, 737, 2

Muzzin, A., Wilson, G., Demarco, R., et al. 2013, ApJ, 767, 39

Noeske, K. G., Faber, S. M., Weiner, B. J., et al. 2007, ApJL, 660, L47

Oliver, S. J., Bock, J., Altieri, B., et al. 2012, MNRAS, 424, 1614

Papovich, C. 2008, ApJ, 676, 206

Papovich, C., Momcheva, I., Willmer, C. N. A., et al. 2010, ApJ, 716, 1503

Papovich, C., Shipley, H. V., Mehrtens, N., et al. 2015, ApJS, submitted

Peng, Y.-j., Lilly, S. J., Kovač, K., et al. 2010, ApJ, 721, 193

Pilbratt, G. L., Riedinger, J. R., Passvogel, T., et al. 2010, A\&A, 518, L1

Poglitsch, A., Waelkens, C., Geis, N., et al. 2010, A\&A, 518, L2

Polletta, M., Tajer, M., Maraschi, L., et al. 2007, ApJ, 663, 81

Rettura, A., Martinez-Manso, J., Stern, D., et al. 2014, ApJ, 797, 109

Rieke, G. H., Young, E. T., Engelbracht, C. W., et al. 2004, ApJS, 154, 25

Rodighiero, G., Cimatti, A., Gruppioni, C., et al. 2010, A\&A, 518, L25

Rodighiero, G., Daddi, E., Baronchelli, I., et al. 2011, ApJL, 739, L40

Rosario, D. J., Santini, P., Lutz, D., et al. 2012, A\&A, 545, A45

Roseboom, I. G., Oliver, S. J., Kunz, M., et al. 2010, MNRAS, 409, 48

Santini, P., Maiolino, R., Magnelli, B., et al. 2014, A\&A, 562, A30

Sawicki, M. 2002, AJ, 124, 3050

Schlegel, D. J., Finkbeiner, D. P., \& Davis, M. 1998, ApJ, 500, 525

Sedgwick, C., Serjeant, S., Pearson, C., et al. 2011, MNRAS, 416, 1862

Sérsic, J. L. 1963, BAAA, 6, 99

Simpson, C., \& Eisenhardt, P. 1999, PASP, 111, 691

Smith, A. J., Wang, L., Oliver, S. J., et al. 2012, MNRAS, 419, 377

Surace, J. A., Shupe, D. L., Fang, F., et al. 2005, BAAS, 37, 63.01

Trenti, M., \& Stiavelli, M. 2008, ApJ, 676, 767

Wang, L., Viero, M., Clarke, C., et al. 2014, MNRAS, 444, 2870

Werner, M. W., Roellig, T. L., Low, F. J., et al. 2004, ApJS, 154, 1

Wuyts, S., Förster Schreiber, N. M., van der Wel, A., et al. 2011, ApJ, 742,96 